\title{
A Numerical Comparison of Inertia-Free Attitude Control Laws for a Spacecraft with a Discrete Flexible Mode
}

\author{
Marc Camblor* \\ Universidad de Sevilla, 41092 Sevilla, Spain \\ Sergio Esteban ${ }^{\S}$ \\ Universidad de Sevilla, 41092 Sevilla, Spain
}

\author{
Antai $\mathrm{Xie}^{\dagger}$ and Gerardo Cruz \\ University of Michigan, 1320 Beal Ave., Ann Arbor MI 48109 \\ Taeyoung Lee ${ }^{\top}$ \\ George Washington University, Washington DC 20052 USA
}

\author{
Dennis S. Bernstein $\|$ \\ University of Michigan, 1320 Beal Ave., Ann Arbor MI 48109
}

\begin{abstract}
Structural flexibility in spacecraft can degrade the accuracy of the attitude control system. With this motivation in mind, we compare the performance of inertia-free attitude control laws for a spacecraft with an undamped discrete flexible mode and demonstrate robustness with respect to unmodeled spacecraft dynamics and actuator saturation. First the equations of motion are derived using Lagrangian dynamics. Next we present the inertia-free control laws considered in the study. Next, we establish a baseline model for subsequent comparisons. Finally, we demonstrate robustness of the control laws to actuator saturation and plant uncertainty through variations in the inertia matrix as well as the parameters of the discrete flexible mode.
\end{abstract}

\section{Introduction}

Attitude control of rigid spacecraft is a widely studied problem. ${ }^{1,2}$ Because of the nonlinear nature of the dynamics and kinematics, nonlinear control methods are required for maneuvers involving high rates and large angles. An additional source of nonlinearity is the type of control actuation, which may involve thrusters with deadzone and on-off behavior, reaction wheels, control-moment gyros, and magnetic torquing. In addition, the representation of spacecraft attitude has many different parameterizations, such as quaternions, Rodrigues parameters, modified Rodrigues parameters, and rotation matrices. ${ }^{1}$ Each type of attitude representation and actuation mechanism has characteristics that impact spacecraft design and attitude control law synthesis.

The assumption that a spacecraft is rigid is an approximation that is limited to low angular velocity maneuvers. Flexible effects become increasingly pronounced, however, as the angular acceleration of the spacecraft increases due to, for example, high-authority, high-bandwidth control. In this case, flexible effects can be included by modeling appendages, such as gravity gradient booms and solar panels, as distributed structures with dynamics governed by partial differential equations. ${ }^{1,3-5}$

The goal of the present paper is to compare the performance of several attitude control laws as applied to flexible spacecraft. In particular, we are interested in the case where the spacecraft inertia and flexible effects are unmodeled in the control law formulation. In the case of a rigid spacecraft, inertia-free control laws are considered in. ${ }^{6-9}$ In the present paper, we investigate the performance of these control laws for flexible spacecraft with unknown inertia as well as flexible components.

Unlike common practice ${ }^{1,3-5}$ in the present paper we do not model flexible effects using continuum mechanics. Instead, we consider a rigid body connected by a spring to a single lumped mass, which moves without damping along

\footnotetext{
* Graduate Student, Department of Aerospace Engineering

${ }^{\dagger}$ Graduate Student, Department of Aerospace Engineering

${ }^{\ddagger}$ Graduate Student, Department of Aerospace Engineering

$\S$ Associate Professor, Department of Aerospace Engineering

I Assistant Professor, Department of Mechanical and Aerospace Engineering

" Professor, Department of Aerospace Engineering
} 
a track that is fixed in the body frame. This idealized and exact model of a flexible spacecraft allows us to carefully compare the performance of inertia-free control laws by varying the inertia of the rigid body, the inertia of the lumped mass, and the stiffness of the spring. The model thus considers the dynamics of a rigid body with a single discrete flexible mode. Additional discrete modes can be included as desired to more fully emulate the dynamics of a flexible spacecraft.

The controllers that we consider include four rotation-matrix-based nonlinear PD and PID control laws, which we designate $\mathrm{SO}(3) / 0, \mathrm{SO}(3) / 3, \mathrm{SO}(3) / 6$, and $\mathrm{SO}(3) / 9$, where the final number indicates the number of integrators in the control law. Loosely speaking, three integrators are used to achieve disturbance rejection with attitude commands, whereas six integrators are used for the inertia estimate. In addition we also consider Retrospective Cost Adaptive Control (RCAC), which applies a retrospective cost least squares algorithm to tune controller gains based on Markov parameters. ${ }^{10-13}$ In this paper we consider rest-to-rest maneuvers (R2R) where the spacecraft is at an initial attitude at rest and the objective is to bring the spacecraft to a specified attitude and at rest. If the R2R maneuver begins from an arbitrary angular velocity, then we use the terminology motion-to-rest (M2R). We consider the case where the actuators can provide continuous torques in $\mathbb{R}^{3}$.

In section II we derive the equations of motion. In section III we derive the $\mathrm{SO}(3)$ control laws and RCAC. Section IV defines the nominal spacecraft parameters. Sections V and VI define baseline tunings for the SO(3) control laws and RCAC for saturation and settling time. Sections VII, VIII and IX study robustness to torque saturation, rigid body inertia, and flexible mode dynamics, respectively.

\section{Equations of Motion}

Consider the dynamics of a spacecraft consisting of a rigid body with a discrete flexible mode modeled as a point mass moving along a fixed slot. Let $M \in \mathbb{R}$ and $J \in \mathbb{R}^{3 \times 3}$ denote the mass and inertia matrix of the spacecraft, respectively. The attitude of the rigid body and the location of its center of mass with respect to an inertial frame are defined as $(R, y) \in \mathrm{SE}(3)$, where $R \in \mathrm{SO}$ (3) transforms of the representation of a vector in the body-fixed frame to its representation in the inertial frame. To represent a discrete flexible mode, a particle with mass $m$ is assumed to be moving along a slot fixed to the rigid body (see Figure 1). The configuration of the slot with respect to the rigid body is described as follows. Let $C$ be the point on the slot whose distance to the center of mass of the rigid body is minimum, and let $\rho \in \mathbb{R}^{3}$ be the vector from the center of mass of the spacecraft body to $C$. The unit vector along the slot is defined as $s \in \mathbb{R}^{3}$. The displacement of the mass along the slot from the point $C$ is denoted by $x \in \mathbb{R}$. The location of the mass is thus given by $\rho_{x} \triangleq \rho+x s \in \mathbb{R}^{3}$ with respect to the body-fixed frame, and $\dot{\rho}_{x}=\dot{x} s$. The point $C$ and the mass are connected by a linear spring with spring constant $\kappa$, and the spring is relaxed when $x=0$. The corresponding configuration manifold is $\mathrm{SE}(3) \times \mathbb{R}$. The attitude kinematics are given by

$$
\dot{R}=R \omega^{\times},
$$

where $\omega \in \mathbb{R}^{3}$ is the angular velocity of the spacecraft resolved in the body frame, and $\omega^{\times}$is the cross-product matrix of $\omega$. Both attitude and rate measurements are assumed to be available. Gyro measurements are assumed to provide measurements of the angular velocity resolved in the spacecraft frame. For simplicity, we assume that gyro measurements are available without noise and without bias. In practice, bias can be corrected by using attitude measurements. Attitude is measured indirectly through direction measurements using sensors such as star trackers. When attitude measurements are given in terms of an alternative attitude representation, such as quaternions, Rodrigues's formula can be used to determine the corresponding rotation matrix. Attitude estimation on $\mathrm{SO}(3)$ is considered in. ${ }^{14}$

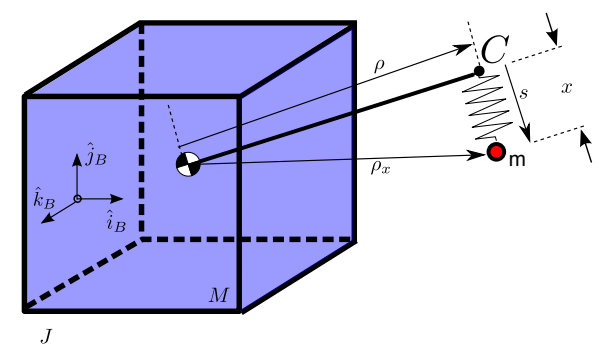

Figure 1: Discrete flexible model of a spacecraft with a lumped mass 


\section{A. Lagrangian Mechanics}

In this section, we derive the equations of motion for a spacecraft with a discrete flexible mode according to Hamilton's variational principle on $\mathrm{SE}(3) \times \mathbb{R}$. Lagrangian mechanics for complex mechanical systems evolving on Lie groups and the two-sphere have been studied in Ref. ${ }^{15,16}$ The corresponding Euler-Lagrange equations are developed in a coordinate-free fashion to avoid singularities associated with local parameterizations. These equations yield concise expressions that are globally defined on the configuration manifold. Here, the Lagrangian of a spacecraft with a discrete flexible mode is formulated, and an action integral is defined. The variation of the action integral yields the Euler-Lagrange equations according to Hamilton's principle, which involves the energy of the complete system, that is, the kinetic and potential energy.

The kinetic energy $E$ of the spacecraft is

$$
E=\frac{1}{2} M\|\dot{y}\|^{2}+\frac{1}{2} \omega^{\mathrm{T}} J \omega
$$

The location of the mass in the inertial-frame is given by

$$
y+R(\rho+x s)=y+R \rho_{x},
$$

and consequently its velocity with respect to the inertial frame is given by

$$
v=\dot{y}+R \dot{x} s+R \omega^{\times} \rho_{x} .
$$

The total kinetic energy, which consists of the kinetic energy of the spacecraft and the point mass, is given by

$$
\begin{aligned}
T & =\frac{1}{2} M\|\dot{y}\|^{2}+\frac{1}{2} \omega^{\mathrm{T}} J \omega+\frac{1}{2} m\left\|\dot{y}+R \dot{x} s+R \omega^{\times} \rho_{x}\right\|^{2} \\
& =\frac{1}{2}(M+m)\|\dot{y}\|^{2}+\frac{1}{2} \omega^{\mathrm{T}}\left(J-m \rho_{x}^{\times 2}\right) \omega+\frac{1}{2} m \dot{x}^{2}+m \dot{y}^{\mathrm{T}}\left[R\left(\dot{x} s+\omega^{\times} \rho_{x}\right)\right]+m \dot{x} s^{\mathrm{T}} \omega^{\times} \rho_{x} .
\end{aligned}
$$

The potential energy, which is due to the linear spring, is given by $U=\frac{1}{2} \kappa x^{2}$. The resulting Lagrangian is

$$
L=\frac{1}{2}(M+m)\|\dot{y}\|^{2}+\frac{1}{2} \omega^{\mathrm{T}}\left[J-m \rho_{x}^{\times 2}\right] \omega+\frac{1}{2} m \dot{x}^{2}+m \dot{y}^{\mathrm{T}}\left[R\left(\dot{x} s+\omega^{\times} \rho_{x}\right)\right]+m \dot{x} s^{\mathrm{T}} \omega^{\times} \rho_{x}-\frac{1}{2} \kappa x^{2} .
$$

The variations of the Lagrangian with respect to $\dot{y}, x, \dot{x}$, and $\omega$ are given by

$$
\begin{aligned}
& \mathbf{D}_{\dot{y}} L^{\mathrm{T}} \delta \dot{y}=\left[(M+m) \dot{y}+m R\left(\dot{x} s+\omega^{\times} \rho_{x}\right)\right]^{\mathrm{T}} \delta \dot{y}, \\
& \mathbf{D}_{x} L^{\mathrm{T}} \delta x=\left[m \dot{y}^{\mathrm{T}} R \omega^{\times} s-m \rho_{x}^{\mathrm{T}} \omega^{\times 2} s-\kappa x\right]^{\mathrm{T}} \delta x, \\
& \mathbf{D}_{\dot{x}} L^{\mathrm{T}} \delta \dot{x}=\left[m \dot{x}+m \dot{y}^{\mathrm{T}} R s+m s^{\mathrm{T}} \omega^{\times} \rho_{x}\right]^{\mathrm{T}} \delta \dot{x}, \\
& \mathbf{D}_{\omega} L^{\mathrm{T}} \delta \omega=\left[\left(J-m \rho_{x}^{\times 2}\right) \omega+m \rho_{x}^{\times} R^{\mathrm{T}} \dot{y}+m \dot{x} \rho_{x}^{\times} s\right]^{\mathrm{T}} \delta \omega,
\end{aligned}
$$

where $\mathbf{D}_{\dot{y}} L$ denotes the derivative of $L$ with respect to $\dot{y}$ and the remaining expressions are defined similarly. An infinitesimal variation of the rotation matrix $R$ can be parametrized by $\eta \in \mathbb{R}^{3}$ as ${ }^{15}$

$$
R^{\mathrm{T}} \delta R=\eta^{\times} .
$$

The variation of $L$ with respect to $R$ can thus be written as

$$
\mathbf{D}_{R} L^{\mathrm{T}} \delta R=\dot{y}^{\mathrm{T}}\left[R \eta^{\times}\left(\dot{x} s+\omega^{\times} \rho_{x}\right)\right]=\left[m\left(\dot{x} s^{\times}+\left(\omega^{\times} \rho_{x}\right)^{-\times}\right) R^{\mathrm{T}} \dot{y}\right]^{\mathrm{T}} \eta .
$$

The corresponding variation of the angular velocity can be obtained as

$$
\delta \omega^{\times}=-\eta^{\times} R^{\mathrm{T}} R \omega^{\times}+R^{\mathrm{T}}\left(R \omega^{\times} \eta^{\times}+R \dot{\eta}^{\times}\right)=\dot{\eta}^{\times}+\omega^{\times} \eta^{\times}-\eta^{\times} \omega^{\times}=\dot{\eta}+\omega^{\times} \eta .
$$

The variation of the action integral $\mathfrak{G} \triangleq \int_{t_{0}}^{t_{f}} L d t$ is then given by

$$
\delta \mathfrak{G}=\int_{t_{0}}^{t_{f}} \mathbf{D}_{\dot{y}} L^{\mathrm{T}} \delta \dot{y}+\mathbf{D}_{\dot{x}} L^{\mathrm{T}} \delta \dot{x}+\mathbf{D}_{x} L^{\mathrm{T}} \delta x+\mathbf{D}_{R} L^{\mathrm{T}} \delta R+\mathbf{D}_{\omega} L^{\mathrm{T}} \delta \omega d t .
$$


Substituting (7)-(13) and using integration by parts yields

$$
\begin{aligned}
\delta \mathfrak{G}=\int_{t_{0}}^{t_{f}} & {\left[-m \ddot{x}-m \ddot{y}^{\mathrm{T}} R s+m s^{\mathrm{T}} \rho_{x}^{\times} \dot{\omega}-m \rho_{x}^{\mathrm{T}} \omega^{\times 2} s-\kappa x\right]^{\mathrm{T}} \delta x } \\
- & {\left[(M+m) \ddot{y}+m \ddot{x} R s+2 m \dot{x} R \omega^{\times} s-m R \rho_{x}^{\times} \dot{\omega}+m R \omega^{\times 2} \rho_{x}\right]^{\mathrm{T}} \delta y } \\
- & {\left[\left(J-m \rho_{x}^{\times 2}\right) \dot{\omega}-m \dot{x}\left(s^{\times} \rho_{x}^{\times}+\rho_{x}^{\times} s^{\times}\right) \omega+m \rho_{x}^{\times} R^{\mathrm{T}} \ddot{y}+m \ddot{x} \rho_{x}^{\times} s\right.} \\
& \left.+\omega^{\times}\left[\left(J-m\left(\rho_{x}^{\times}\right)^{\mathrm{T}}\right) \omega+m \dot{x} \rho_{x}^{\times} s\right]\right]^{\mathrm{T}} \eta d t .
\end{aligned}
$$

According to Hamilton's principle $\delta \mathfrak{G}=0$ for all $\delta x, \delta y$, and $\eta$. Hence the coefficients of $\delta x, \delta y$ and $\eta$ in (15) are zero, which implies the Euler-Lagrange equations

$$
\begin{gathered}
m \ddot{x}+m \ddot{y}^{\mathrm{T}} R s-m s^{\mathrm{T}} \rho_{x}^{\times} \dot{\omega}+m \rho_{x}^{\mathrm{T}} \omega^{\times 2} s+\kappa x=0, \\
(M+m) \ddot{y}+m \ddot{x} R s+2 m \dot{x} R \omega^{\times} s-m R \rho_{x}^{\times} \dot{\omega}+m R \omega^{\times 2} \rho_{x}=0, \\
\left(J-m \rho_{x}^{\times 2}\right) \dot{\omega}-m \dot{x}\left(s^{\times} \rho_{x}^{\times}+\rho_{x}^{\times} s^{\times}\right) \omega+m \rho_{x}^{\times} R^{\mathrm{T}} \ddot{y}+m \ddot{x} \rho_{x}^{\times} s+\omega^{\times}\left[\left(J-m \rho_{x}^{\times 2}\right) \omega+m \dot{x} \rho_{x}^{\times} s\right]=B u+z_{\text {dist }},
\end{gathered}
$$

where $B \in \mathbb{R}^{3 \times 3}$ is the torque input matrix, $u=\left[\begin{array}{lll}u_{1} & u_{2} & u_{3}\end{array}\right]^{\mathrm{T}}$ is the control torque input, and $z_{\text {dist }}$ represents external disturbances. We rewrite (16), (17) and (18) in matrix form as

$$
\left[\begin{array}{ccc}
m & m s^{\mathrm{T}} R^{\mathrm{T}} & -m s^{\mathrm{T}} \rho_{x}^{\times} \\
m R s & (M+m) I & -m R \rho_{x}^{\times} \\
m \rho_{x}^{\times} s & m \rho_{x}^{\times} R^{\mathrm{T}} & J-m \rho_{x}^{\times 2}
\end{array}\right]\left[\begin{array}{c}
\ddot{x} \\
\ddot{y} \\
\dot{\omega}
\end{array}\right]=\left[\begin{array}{c}
-m \rho_{x}^{\mathrm{T}} \omega^{\times 2} s-\kappa x \\
-2 m \dot{x} R \omega^{\times} s-m R \omega^{\times 2} \rho_{x} \\
m \dot{x}\left(s^{\times} \rho_{x}^{\times}+\rho_{x}^{\times} s^{\times}\right) \omega-\omega^{\times}\left[\left(J-m \rho_{x}^{\times 2}\right) \omega+m \dot{x} \rho_{x}^{\times} s\right]+B u+z_{\text {dist }}
\end{array}\right] .
$$

As a special case, we can consider the dynamics in $\mathrm{SO}(3) \times \mathbb{R}$ by redefining the total kinetic energy of the system without translational terms as

$$
T=\frac{1}{2} \omega^{\mathrm{T}} J \omega+\frac{1}{2} m\left\|R \dot{x} s+R \omega^{\times} \rho_{x}\right\|^{2} .
$$

This is also equivalent to setting $\ddot{y}=0$ in (17) and (18) recovering the equations in $\mathrm{SO}(3)$, which have the form

$$
\begin{gathered}
m \ddot{x}-m s^{\mathrm{T}} \rho_{x}^{\times} \dot{\omega}+m \rho_{x}^{\mathrm{T}} \omega^{\times 2} s+\kappa x=0, \\
\left(J-m \rho_{x}^{\times 2}\right) \dot{\omega}-m \dot{x}\left(s^{\times} \rho_{x}^{\times}+\rho_{x}^{\times} s^{\times}\right) \omega+m \ddot{x} \rho_{x}^{\times} s+\omega^{\times}\left[\left(J-m \rho_{x}^{\times 2}\right) \omega+m \dot{x} \rho_{x}^{\times} s\right]=B u+z_{\text {dist }} .
\end{gathered}
$$

Equations (20) and (21) are used in the remainder of the paper to study the performance of attitude control laws with an unmodeled discrete flexible mode.

\section{Controller Construction}

In this section we describe four $\mathrm{SO}(3)$ control laws along with RCAC. The controllers are derived for use with continuously variable thruster actuation without onboard momentum storage. These algorithms are inertia-free control laws whose goal is to bring the spacecraft to a prescribed attitude without knowledge of the spacecraft inertia.

\section{A. $\mathrm{SO}(3) / 0$}

The $\mathrm{SO}(3) / 0$ control law for almost global stabilization ${ }^{17,18}$ is given by

$$
u=-B^{-1}\left(K_{\mathrm{p}} S+K_{\mathrm{v}} \omega\right),
$$

where $K_{\mathrm{p}} \in \mathbb{R}$ and $K_{\mathrm{v}} \in \mathbb{R}^{3 \times 3}$ are proportional (attitude) and derivative (angular velocity) positive-definite gains, respectively. The attitude error $S$ is defined by

$$
S \triangleq \sum_{i=1}^{3} a_{i}\left(\tilde{R}^{\mathrm{T}} e_{i}\right) \times e_{i}
$$


where $a_{1}, a_{2}, a_{3}$ are positive numbers, $e_{1}, e_{2}, e_{3}$ are the standard basis vectors, and the rotation matrix $\tilde{R}=R R_{\mathrm{d}}^{\mathrm{T}}$ represents the pointing error between the current attitude $R$ and the desired attitude $R_{\mathrm{d}}$. Note that the control law (22) is inertia-free. We use the following Lemma to show that $V$ is a Lyapunov function.

Lemma 1. ${ }^{18}$ Let $a_{1}, a_{2}, a_{3}$ be distinct and let $R$ be a rotation matrix. Then:

i) For all $i, j=1,2,3, R_{i j} \in[-1,1]$.

ii) $\operatorname{tr}(A-A R) \geq 0$.

iii) $\operatorname{tr}(A-A R)=0$ if and only if $R=I$.

Defining $A=\operatorname{diag}\left(a_{1}, a_{2}, a_{3}\right)$, the effect of the control law (22) on the attitude of a rigid spacecraft follows from the Lyapunov function

$$
V(\omega, \tilde{R}) \triangleq \frac{1}{2} \omega^{\mathrm{T}} J \omega+K_{\mathrm{p}} \operatorname{tr}(A-A \tilde{R}),
$$

for which $\dot{V}(\omega, \tilde{R})=-\omega^{\mathrm{T}} K_{\mathrm{v}} \omega$.

By choosing $K_{\mathrm{v}}$ to be a function of $\omega$, the control law (22) satisfies the following saturation bounds. ${ }^{18,19}$ Let $\sigma_{\min }(B)$ denote the minimum singular value of $B$.

Proposition 1. Let $\alpha$ and $\beta$ be positive numbers, let $A=\operatorname{diag}\left(a_{1}, a_{2}, a_{3}\right)$ have distinct positive diagonal entries, and let $K_{\mathrm{p}}$ and $K_{\mathrm{v}}(\omega)$ be given by

$$
K_{\mathrm{p}}=\frac{\alpha}{\operatorname{tr} A}
$$

and

$$
K_{\mathrm{v}}(\omega)=\beta\left[\begin{array}{ccc}
\frac{1}{1+\left|\omega_{1}\right|} & 0 & 0 \\
0 & \frac{1}{1+\left|\omega_{2}\right|} & 0 \\
0 & 0 & \frac{1}{1+\left|\omega_{3}\right|}
\end{array}\right] .
$$

Then, for all $t \geq 0$, the control torque given by (22) satisfies

$$
\|u(t)\|_{\infty} \leq \frac{\alpha+\beta}{\sigma_{\min }(B)} .
$$

Alternative forms of the gain $K_{\mathrm{v}}(\omega)$ are given in. ${ }^{20}$

\section{B. $\mathrm{SO}(3) / 3$}

We include integral action by extending (22) to the $\mathrm{SO}(3) / 3$ control law

$$
u=-B^{-1}\left[K_{\mathrm{p}} S+K_{\mathrm{v}} K_{1} S+K_{\mathrm{i}} \bar{C}_{\mathrm{d}} D^{-1} \bar{C}_{\mathrm{d}}^{\mathrm{T}} \int_{0}^{t}\left[\tilde{\omega}(s)+K_{1} S(s)\right] \mathrm{d} s+K_{\mathrm{v}} \tilde{\omega}\right],
$$

where $K_{\mathrm{i}} \in \mathbb{R}, K_{1} \in \mathbb{R}^{3 \times 3}$ are positive definite, $\bar{C}_{\mathrm{d}} \in \mathbb{R}^{3 \times 3}$, and $D \in \mathbb{R}^{3 \times 3}$ is positive definite. The angular-velocity error $\tilde{\omega}$ is defined by $\tilde{\omega} \triangleq \omega-\tilde{R}^{\mathrm{T}} \omega_{\mathrm{d}}$, where $\omega_{\mathrm{d}}$ is the desired possibly time-varying angular velocity. The control law (28) is suggested by the $\mathrm{SO}(3) / 9$ control law (35) given below by specializing $u=B^{-1}\left(v_{2}+v_{3}\right)$. Although $\mathrm{SO}(3) / 3$ does not have a known Lyapunov function that ensures closed-loop stability, simulation results suggest that it is stabilizing for all gains $K_{\mathrm{p}}, K_{\mathrm{v}}$, and $K_{\mathrm{i}}$.

\section{C. $\mathrm{SO}(3) / 6$}

The $\mathrm{SO}(3) / 6$ control law is a simplification of the $\mathrm{SO}(3) / 9$ control law given below by specializing $u=B^{-1}\left(v_{1}+\right.$ $\left.v_{3}\right)$ in (35), and $A_{\mathrm{d}}=0$ in (31) and (32). In particular, this control law has the form

$$
\left.u=-B^{-1}\left[K_{\mathrm{p}} S+K_{\mathrm{v}} K_{1} S++K_{1} S(s)\right)+K_{\mathrm{v}} \tilde{\omega}+\hat{J} K_{1} \dot{S}+(\hat{J} \omega) \times \omega\right],
$$

where $\hat{J}$ is the inertia estimate updated by (30), (32), and $\dot{S}$ is updated by (33). 


\section{D. $\mathrm{SO}(3) / 9$} by

To develop an estimate of the spacecraft inertia, we introduce the notation $J \omega=L(\omega) \gamma$, where $\gamma \in \mathbb{R}^{6}$ is defined

$$
\gamma \triangleq\left[\begin{array}{llllll}
J_{11} & J_{22} & J_{33} & J_{23} & J_{13} & J_{12}
\end{array}\right]^{\mathrm{T}}
$$

and

$$
L(\omega) \triangleq\left[\begin{array}{cccccc}
\omega_{1} & 0 & 0 & 0 & \omega_{3} & \omega_{2} \\
0 & \omega_{2} & 0 & \omega_{3} & 0 & \omega_{1} \\
0 & 0 & \omega_{3} & \omega_{2} & \omega_{1} & 0
\end{array}\right]
$$

Next, let $\hat{J} \in \mathbb{R}^{3 \times 3}$ denote an estimate of $J$, and define the inertia-estimation error $\tilde{J} \triangleq J-\hat{J}$. Letting $\hat{\gamma}, \tilde{\gamma} \in \mathbb{R}^{6}$ represent $\hat{J}, \tilde{J}$, respectively, it follows that $\tilde{\gamma}=\gamma-\hat{\gamma}$. Likewise, let $\hat{z}_{\text {dist }} \in \mathbb{R}^{3}$ denote an estimate of $z_{\text {dist }}$, and define the disturbance-estimation error $\tilde{z}_{\text {dist }} \triangleq z_{\text {dist }}-\hat{z}_{\text {dist }}$.

Assuming that the disturbance is harmonic with known spectrum, it follows that $z_{\text {dist }}$ can be modeled by

$$
\dot{d}=A_{\mathrm{d}} d, \quad z_{\text {dist }}=C_{\mathrm{d}} d,
$$

where $A_{\mathrm{d}} \in \mathbb{R}^{n_{\mathrm{d}} \times n_{\mathrm{d}}}$ and $C_{\mathrm{d}} \in \mathbb{R}^{3 \times n_{\mathrm{d}}}$ are known matrices. In this model, $d(0)$ is unknown, which is equivalent to the assumption that the amplitude and phase of all harmonic components in the disturbance are unknown; however, the spectrum of $d$ is assumed to be known. To provide asymptotic rejection of harmonic disturbances, the matrix $A_{\mathrm{d}}$ is chosen to include eigenvalues of all frequency components that may be present in $z_{\text {dist }}$, where the zero eigenvalue corresponds to constant disturbances. Since $z_{\text {dist }}$ is harmonic, $A_{\mathrm{d}}$ is chosen to be skew symmetric. Let $\hat{d} \in \mathbb{R}^{n_{\mathrm{d}}}$ denote an estimate of $d$, and define the disturbance-state estimation error $\tilde{d} \triangleq d-\hat{d}$.

Theorem 1. Let $K_{\mathrm{p}} \in \mathbb{R}$ be positive, let $K_{1} \in \mathbb{R}^{3 \times 3}$, let $Q \in \mathbb{R}^{6 \times 6}$ and $D \in \mathbb{R}^{n_{\mathrm{d}} \times n_{\mathrm{d}}}$ be positive definite, let $A=\operatorname{diag}\left(a_{1}, a_{2}, a_{3}\right)$ be a diagonal positive-definite matrix with distinct diagonal entries, and define the attitude error $S$ by (23). Then the Lyapunov candidate

$$
V(\tilde{\omega}, \tilde{R}, \tilde{\gamma}, \tilde{d}) \triangleq \frac{1}{2}\left(\tilde{\omega}+K_{1} S\right)^{\mathrm{T}} J\left(\tilde{\omega}+K_{1} S\right)+K_{\mathrm{p}} \operatorname{tr}(A-A \tilde{R})+\frac{1}{2} \tilde{\gamma}^{\mathrm{T}} Q \tilde{\gamma}+\frac{1}{2} \tilde{d}^{\mathrm{T}} D \tilde{d}
$$

is positive definite, that is, $V$ is nonnegative, and $V=0$ if and only if $\tilde{\omega}=0, \tilde{R}=I, \tilde{\gamma}=0$, and $\tilde{d}=0$.

Theorem 2. Let $K_{\mathrm{p}} \in \mathbb{R}, K_{\mathrm{v}} \in \mathbb{R}^{3 \times 3}, K_{1} \in \mathbb{R}^{3 \times 3}, Q \in \mathbb{R}^{6 \times 6}$, and $D \in \mathbb{R}^{n_{\mathrm{d}} \times n_{\mathrm{d}}}$ be positive definite, assume that $A_{\mathrm{d}}^{\mathrm{T}} D+D A_{\mathrm{d}}$ is negative semidefinite, let $A=\operatorname{diag}\left(a_{1}, a_{2}, a_{3}\right)$ be a diagonal positive-definite matrix with distinct diagonal entries, define $S$ and $V$ as in Theorem 1, and let $\hat{\gamma}$ and $\hat{d}$ satisfy

$$
\dot{\hat{\gamma}}=Q^{-1}\left[L^{\mathrm{T}}(\omega) \omega^{\times}+L^{\mathrm{T}}\left(K_{1} \dot{S}+\tilde{\omega} \times \omega-\tilde{R}^{\mathrm{T}} \dot{\omega}_{\mathrm{d}}\right)\right]\left(\tilde{\omega}+K_{1} S\right)
$$

where

$$
\dot{S}=\sum_{i=1}^{3} a_{i}\left[\left(\tilde{R}^{\mathrm{T}} e_{i}\right) \times \tilde{\omega}\right] \times e_{i}
$$

and

$$
\dot{\hat{d}}=A_{\mathrm{d}} \hat{d}+D^{-1} C_{\mathrm{d}}^{\mathrm{T}}\left(\tilde{\omega}+K_{1} S\right), \quad \hat{z}_{\mathrm{dist}}=C_{\mathrm{d}} \hat{d} .
$$

Furthermore, let

$$
u=B^{-1}\left(v_{1}+v_{2}+v_{3}\right),
$$

where

$$
v_{1} \triangleq-(\hat{J} \omega) \times \omega-\hat{J}\left(K_{1} \dot{S}+\tilde{\omega} \times \omega-\tilde{R}^{\mathrm{T}} \dot{\omega}_{\mathrm{d}}\right)
$$




$$
\begin{gathered}
v_{2} \triangleq-\hat{z}_{\text {dist }}, \\
v_{3} \triangleq-K_{\mathrm{v}}\left(\tilde{\omega}+K_{1} S\right)-K_{\mathrm{p}} S .
\end{gathered}
$$

Then,

$$
\dot{V}(\tilde{\omega}, \tilde{R}, \tilde{\gamma}, \tilde{d})=-\left(\tilde{\omega}+K_{1} S\right)^{\mathrm{T}} K_{\mathrm{v}}\left(\tilde{\omega}+K_{1} S\right)-K_{\mathrm{p}} S^{\mathrm{T}} K_{1} S+\frac{1}{2} \tilde{d}^{\mathrm{T}}\left(A_{\mathrm{d}}^{\mathrm{T}} D+D A_{\mathrm{d}}\right) \tilde{d}
$$

is negative semidefinite. Equation (21) can be rewritten in terms of $\tilde{\omega}$ as

$$
\begin{aligned}
\left(J-m \rho_{x}^{\times 2}\right) \dot{\tilde{\omega}}= & m \dot{x}\left(s^{\times} \rho_{x}^{\times}+\rho_{x}^{\times} s^{\times}\right)\left(\tilde{\omega}+\tilde{R}^{\mathrm{T}} \omega_{\mathrm{d}}\right)-m \ddot{x} \rho_{x}^{\times} s \\
& -\left(\tilde{\omega}+\tilde{R}^{\mathrm{T}} \omega_{\mathrm{d}}\right)^{\times}\left[\left(J-m \rho_{x}^{\times 2}\right)\left(\tilde{\omega}-\tilde{R}^{\mathrm{T}} \omega_{\mathrm{d}}\right)+m \dot{x} \rho_{x}^{\times} s\right]+B u+z_{\text {dist }} .
\end{aligned}
$$

Furthermore, the equilibrium manifold $(\tilde{\omega}, \tilde{R},(\tilde{\gamma}, \tilde{d}))=\left(0, I, \mathcal{Q}_{0}\right)$ of the closed-loop system given by (32)-(34) and (40) is locally asymptotically stable, and the remaining equilibrium manifolds given by $\left(0, \mathcal{R}_{i}, \mathcal{Q}_{i}\right)$, for $i \in\{1,2,3\}$ are unstable. Finally, the set of all initial conditions converging to these equilibrium manifolds forms a lower dimensional submanifold of $\mathbb{R}^{3} \times \mathrm{SO}(3) \times \mathbb{R}^{6} \times \mathbb{R}^{3}$.

\section{E. RCAC}

In this section, we review the cumulative retrospective cost adaptive controller developed in ${ }^{11,21-23}$ for linear plants. First, consider the MIMO discrete-time system

$$
\begin{aligned}
x(k+1) & =A x(k)+B u(k), \\
y_{0}(k) & =E_{1} x(k), \\
z(k) & =r(k)-y_{0}(k),
\end{aligned}
$$

where $x(k) \in \mathbb{R}^{n}, z(k) \in \mathbb{R}^{l_{z}}, u(k) \in \mathbb{R}^{l_{u}}, r(k) \in \mathbb{R}^{l_{w}}$, and $k \geq 0$. The goal is to develop an adaptive output feedback controller that minimizes the command-following error $z$ in the presence of the command signal $r$ with minimal modeling information about the dynamics and $r$.

We represent (41) and (43) as the time-series model from $u$ to $z$ given by

$$
z(k)=E_{1} A^{m} x(k-m)-E_{0} r(k)+\bar{H} \bar{U}(k-1),
$$

where $k>m, \bar{H}$ is defined by

$$
\bar{H} \triangleq\left[\begin{array}{ccc}
H_{1} & \cdots & H_{m}
\end{array}\right] \in \mathbb{R}^{l_{z} \times m l_{u}},
$$

where $H_{i} \triangleq E_{1} A^{i-1} B$ are the Markov parameters, and

$$
\bar{U}(k-1) \triangleq\left[\begin{array}{c}
u(k-1) \\
\vdots \\
u(k-m)
\end{array}\right] \in \mathbb{R}^{m l_{u}}
$$

is the extended control vector.

Next, we present an adaptive control algorithm for the general control problem represented by (41)-(43). The control $u(k)$ is given by the strictly proper time-series controller of order $n_{\mathrm{c}}$ of the form

$$
u(k)=\sum_{i=1}^{n_{\mathrm{c}}} M_{i}(k) u(k-i)+\sum_{i=1}^{n_{\mathrm{c}}} N_{i}(k) z(k-i),
$$


where, for all $i=1, \ldots, n_{\mathrm{c}}, M_{i}(k) \in \mathbb{R}^{l_{u} \times l_{u}}$ and $N_{i}(k) \in \mathbb{R}^{l_{u} \times l_{z}}$. The control (45) can be expressed as

$$
u(k)=\theta(k) \phi(k-1),
$$

where

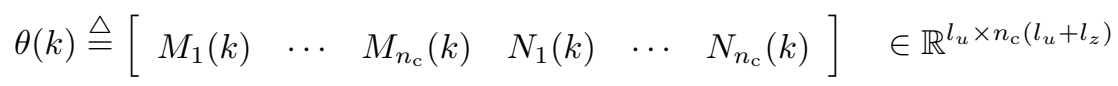

is the controller gain matrix, and the regressor vector $\phi(k-1)$ is given by

$$
\phi(k-1) \triangleq\left[\begin{array}{c}
u(k-1) \\
\vdots \\
u\left(k-n_{\mathrm{c}}\right) \\
z(k-1) \\
\vdots \\
z\left(k-n_{\mathrm{c}}\right)
\end{array}\right] \in \mathbb{R}^{n_{\mathrm{c}}\left(l_{u}+l_{z}\right)} .
$$

Next, we define the retrospective performance

$$
\hat{z}\left(k-k_{j}\right) \triangleq \mathcal{S}_{j}\left(k-k_{j}\right)+\mathcal{H}_{j} \hat{U}_{j}\left(k-k_{j}-1\right),
$$

where $\mathcal{S}(k) \triangleq E_{1} A^{m} x(k-m)-E_{0} r(k)+\mathcal{H}^{\prime} U^{\prime}(k-1)$, and the past controls $U_{j}\left(k-k_{j}-1\right)$ are replaced by the surrogate controls $\hat{U}_{j}\left(k-k_{j}-1\right)$. Now, we express the extended retrospective performance as

$$
\hat{Z}(k) \triangleq\left[\begin{array}{c}
\hat{z}\left(k-k_{1}\right) \\
\vdots \\
\hat{z}\left(k-k_{s}\right)
\end{array}\right] \in \mathbb{R}^{s l_{z}},
$$

which can be rewritten as

$$
\hat{Z}(k)=\tilde{\mathcal{S}}(k)+\tilde{\mathcal{H}} \hat{\tilde{U}}(k-1),
$$

where the components of $\hat{\tilde{U}}(k-1) \in \mathbb{R}^{l} \tilde{U}$ are the components of $\hat{U}_{1}\left(k-k_{1}-1\right), \ldots, \hat{U}_{s}\left(k-k_{s}-1\right)$ ordered in the same way as the components of $\tilde{U}(k-1)$. Hence

$$
\hat{Z}(k)=Z(k)-\tilde{\mathcal{H}} \tilde{U}(k-1)+\tilde{\mathcal{H}} \hat{\tilde{U}}(k-1) .
$$

Finally, we define the retrospective cost function

$$
J(\hat{\tilde{U}}(k-1), k) \triangleq \hat{Z}^{\mathrm{T}}(k) R(k) \hat{Z}(k),
$$

where $R(k) \in \mathbb{R}^{l_{z} s \times l_{z} s}$ is a positive-definite performance weighting. To ensure that (51) has a global minimizer, we consider the regularized cost

$$
\bar{J}(\hat{\tilde{U}}(k-1), k) \triangleq \hat{Z}^{\mathrm{T}}(k) R(k) \hat{Z}(k)+\eta(k) \hat{\tilde{U}}^{\mathrm{T}}(k-1) \hat{\tilde{U}}(k-1),
$$

where $\eta(k) \geq 0$. Substituting (50) into (52) yields

$$
\bar{J}(\hat{\tilde{U}}(k-1), k)=\hat{\tilde{U}}(k-1)^{\mathrm{T}} \mathcal{A}(k) \hat{\tilde{U}}(k-1)+\mathcal{B}(k) \hat{\tilde{U}}(k-1)+\mathcal{C}(k),
$$

where

$$
\begin{aligned}
& \mathcal{A}(k) \triangleq \tilde{\mathcal{H}}^{\mathrm{T}} R(k) \tilde{\mathcal{H}}+\eta(k) I_{l_{\tilde{U}}}, \\
& \mathcal{B}(k) \triangleq 2 \tilde{\mathcal{H}}^{\mathrm{T}} R(k)[Z(k)-\tilde{\mathcal{H}} \tilde{U}(k-1)], \\
& \mathcal{C}(k) \triangleq Z^{\mathrm{T}}(k) R(k) Z(k)-2 Z^{\mathrm{T}}(k) R(k) \tilde{\mathcal{H}} \tilde{U}(k-1)+\tilde{U}^{\mathrm{T}}(k-1) \tilde{\mathcal{H}}^{\mathrm{T}} R(k) \tilde{\mathcal{H}} \tilde{U}(k-1) .
\end{aligned}
$$


If either $\tilde{\mathcal{H}}$ has full column rank or $\eta(k)>0$, then $\mathcal{A}(k)$ is positive definite. In this case, $\bar{J}(\hat{\tilde{U}}(k-1), k)$ has the unique global minimizer given by

$$
\hat{\tilde{U}}(k-1)=-\frac{1}{2} \mathcal{A}^{-1}(k) \mathcal{B}(k) .
$$

Next, let $q$ be a positive integer such that $\tilde{U}(k-1)$ contains $u(k-q)$ and define the cumulative cost function as

$$
J_{\mathrm{R}}(\theta(k)) \triangleq \sum_{i=1}^{k} \lambda^{k-i}\left\|\phi^{\mathrm{T}}(k-q-1) \theta^{\mathrm{T}}(k-1)-\hat{u}^{\mathrm{T}}(k-q)\right\|^{2},
$$

where $\|\cdot\|$ is the Euclidean norm, and $\lambda \in(0,1]$ is the forgetting factor. Minimizing (54) yields

$$
\begin{aligned}
\theta^{\mathrm{T}}(k)= & \theta^{\mathrm{T}}(k-1)+P(k-1) \phi(k-q-1)\left[\phi^{\mathrm{T}}(k-q) P(k-1) \phi(k-q-1)+\lambda\right]^{-1} \\
& {\left[\phi^{\mathrm{T}}(k-q-1) \theta^{\mathrm{T}}(k-1)-\hat{u}^{\mathrm{T}}(k-q)\right] . }
\end{aligned}
$$

The error covariance is updated by

$$
\begin{aligned}
P(k)= & \lambda^{-1} P(k-1)-\lambda^{-1} P(k-1) \phi(k-q-1) \\
& {\left[\phi^{\mathrm{T}}(k-q-1) P(k-1) \phi(k-q)+\lambda(k)\right]^{-1} \phi^{\mathrm{T}}(k-q-1) P(k-1) . }
\end{aligned}
$$

We initialize the error covariance matrix as $P(0)=\alpha(k) I$, where $\alpha(k)>0$.

\section{Nominal Spacecraft Parameters}

In this section we define a nominal model of a spacecraft with a discrete flexible mode. We set $\rho_{x}=\left[\begin{array}{lll}1 & 0 & 0\end{array}\right]^{\mathrm{T}} \mathrm{m}$, and $s=\left[\begin{array}{lll}0 & 1 & 0\end{array}\right]^{\mathrm{T}} \mathrm{m}$. Thus the lumped mass moves along the $\hat{\jmath}$ body-frame direction, offset $1 \mathrm{~m}$ from the spacecraft body-frame origin.

The lumped mass and spring stiffness are, respectively, $m=3 \mathrm{~kg}$ and $k=2 \mathrm{~N}-\mathrm{m}$, the spacecraft mass is $M=60$ $\mathrm{kg}$, and the spacecraft inertia is given by

$$
J=\left[\begin{array}{ccc}
30 & 0 & 0 \\
0 & 25 & 0 \\
0 & 0 & 15
\end{array}\right] .
$$

Let the spacecraft be initially at rest with the initial attitude and angular velocity $R_{0}=I$ and $\omega_{0}=\left[\begin{array}{ll}0 & 0\end{array}\right]^{\mathrm{T}} \mathrm{rad} / \mathrm{sec}$. The goal is to bring the spacecraft to rest at the desired attitude $R_{\mathrm{d}}$ and angular velocity $\omega_{\mathrm{d}}=\left[\begin{array}{ll}0 & 0\end{array}\right]^{\mathrm{T}}$. We simulate a rest-to-rest (R2R) scenario commanding a 60-deg rotation about the body-fixed direction $n=\frac{1}{\sqrt{3}}\left[\begin{array}{lll}1 & 1 & 1\end{array}\right]^{\mathrm{T}}$. The desired attitude is obtained by using Rodrigues's rotation formula

$$
R_{\mathrm{d}}=I \cos (\theta)+(1-\cos (\theta)) n n^{\mathrm{T}}+\sin (\theta) n^{\times} .
$$

Hence, the desired attitude matrix is given by

$$
R_{\mathrm{d}}=\left[\begin{array}{ccc}
0.6667 & -0.3333 & 0.6667 \\
0.6667 & 0.6667 & -0.3333 \\
-0.3333 & 0.6667 & 0.6667
\end{array}\right]
$$

We assume that the spacecraft is fully actuated (that is, $B=I$ ).

To evaluate performance for the R2R maneuvers, we define the settling-time metric

$$
k_{0}=\min _{h>100}\left\{h: \text { for all } i \in\{1, \ldots, 100\}, e\left((h-i) T_{\mathrm{s}}\right)<0.05 \mathrm{rad}\right\},
$$

where $h$ is the simulation step, $T_{\mathrm{s}}$ is the integration step size, and $e\left(k T_{\mathrm{s}}\right)$ is the eigenaxis attitude error at the $k$ th simulation step given by

$$
e\left(k T_{\mathrm{s}}\right)=\cos ^{-1}\left(\frac{1}{2}\left[\operatorname{tr} \tilde{R}\left(k T_{\mathrm{s}}\right)-1\right]\right)
$$




\section{Baseline Tuning for the SO(3) Control Laws for Saturation and Settling time}

We define the controller parameters $\alpha=1, \beta=1, K_{\mathrm{i}}=0.015, K_{1}=I$, and $A=\operatorname{diag}(1,2,3)$. These parameters are identical and constant for all four $\mathrm{SO}(3)$ controllers. The following subsections show the $\mathrm{SO}(3)$ control laws tuned for actuator saturation. Note that the commanded maneuver is about a non-principal axis.

\section{A. Baseline Tuning for the SO(3)/0 Control Law}

Figure 2 shows the $\mathrm{SO}(3) / 0$ control law with actuator saturation. The saturation level is chosen to obtain a settling time of about $500 \mathrm{sec}$.

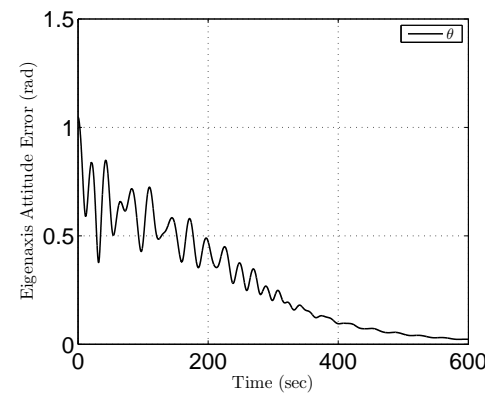

(a) Eigenaxis attitude error.

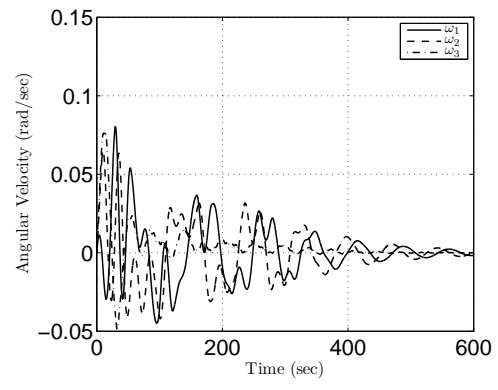

(b) Angular velocity components.

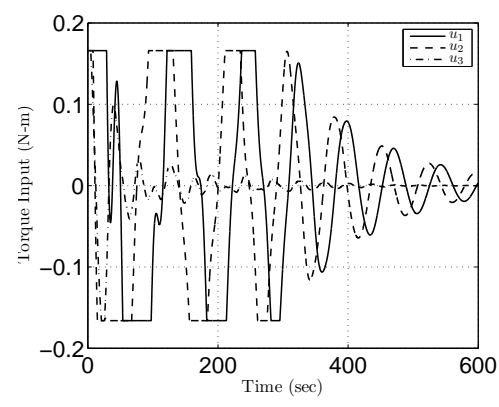

(c) Torque input.

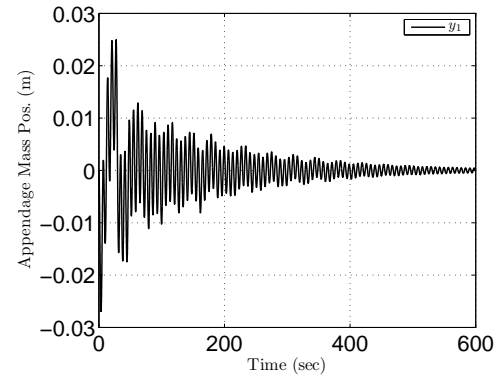

(d) Lumped mass position.

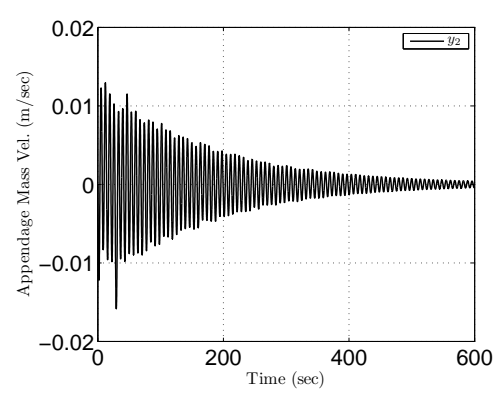

(e) Lumped mass velocity.

Figure 2: $\mathrm{R} 2 \mathrm{R}$ for the $\mathrm{SO}(3) / 0$ control law with actuator saturation of $0.16 \mathrm{~N}-\mathrm{m}$. The maneuver is a $60-\mathrm{deg}$ rotation about the body-fixed frame direction $n=\frac{1}{\sqrt{3}}\left[\begin{array}{lll}1 & 1 & 1\end{array}\right]^{\mathrm{T}}$. The spacecraft reaches the desired attitude, and the lumped mass is brought to rest with a settling time of $505.3 \mathrm{sec}$.

\section{B. Baseline Tuning for the SO(3)/3 Control Law}

Figure 3 shows the $\mathrm{SO}(3) / 3$ control law with actuator saturation. The saturation level is chosen to obtain a settling time of about $500 \mathrm{sec}$. 


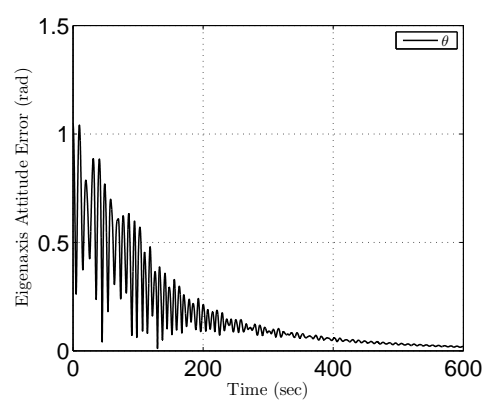

(a) Eigenaxis attitude error.

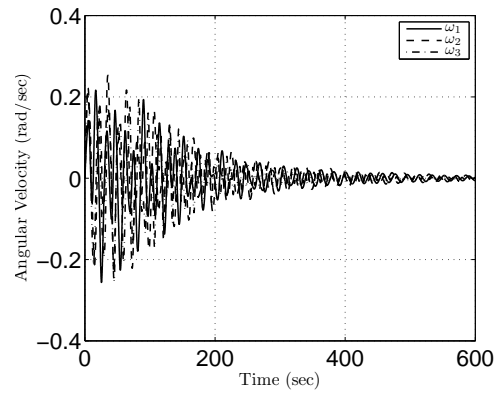

(b) Angular velocity components.

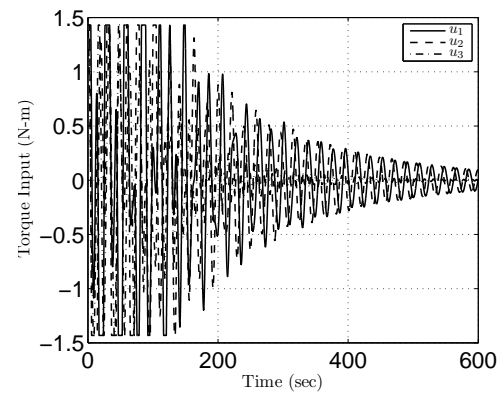

(c) Torque input.

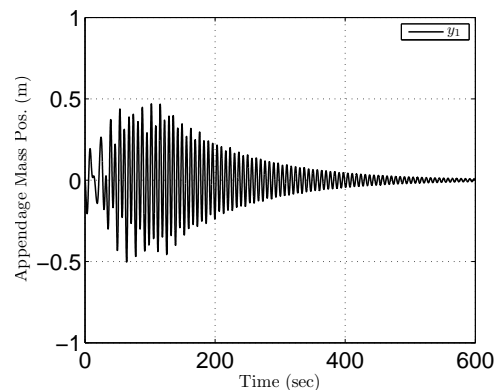

(d) Lumped mass position.

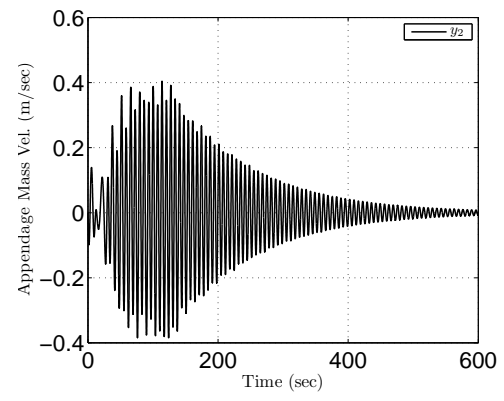

(e) Lumped mass velocity.

Figure 3: R2R for the $\mathrm{SO}(3) / 3$ controller with actuator saturation of $1.43 \mathrm{~N}-\mathrm{m}$. The maneuver is a 60 -deg rotation about the body-fixed frame direction $n=\frac{1}{\sqrt{3}}\left[\begin{array}{lll}1 & 1 & 1\end{array}\right]^{\mathrm{T}}$. The spacecraft reaches the desired attitude, and the lumped mass is brought to rest with a settling time of $482.2 \mathrm{sec}$.

\section{Baseline Tuning for the $S O(3) / 6$ Control Law}

Figure 4 shows the $\mathrm{SO}(3) / 6$ control law with actuator saturation. The saturation level is chosen to obtain a settling time of about $100 \mathrm{sec}$. 


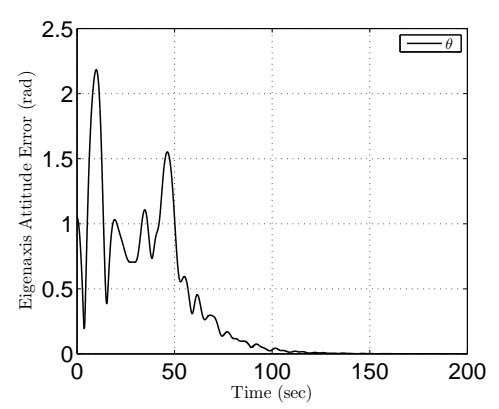

(a) Eigenaxis attitude error.

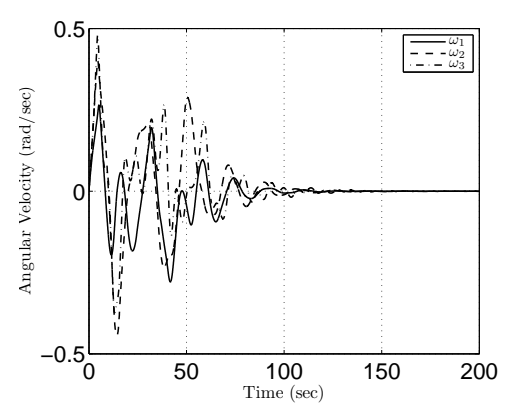

(b) Angular velocity components.

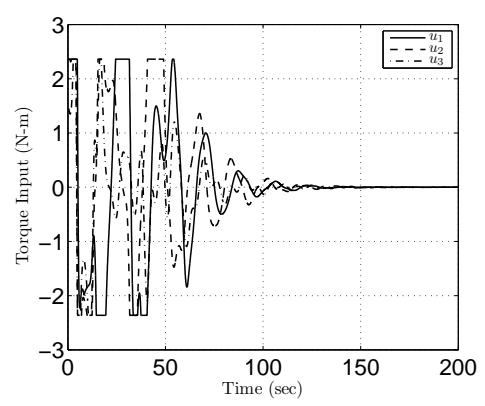

(c) Torque input.

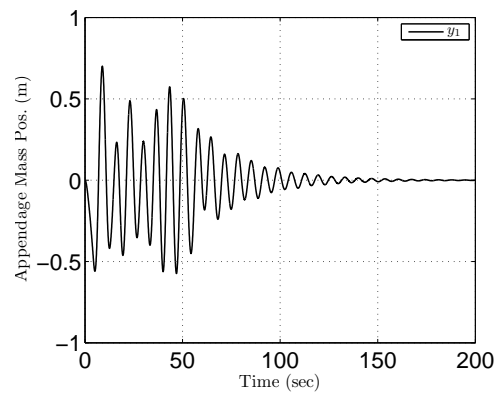

(d) Lumped mass position.

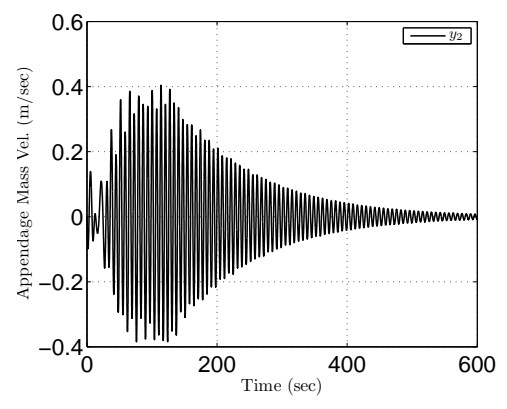

(e) Lumped mass velocity.

Figure 4: R2R for the $\mathrm{SO}(3) / 6$ controller with actuator saturation of $2.36 \mathrm{~N}-\mathrm{m}$. The maneuver is a 60 -deg rotation about the body-fixed frame direction $n=\frac{1}{\sqrt{3}}\left[\begin{array}{lll}1 & 1 & 1\end{array}\right]^{\mathrm{T}}$. The spacecraft reaches the desired attitude, and the lumped mass is brought to rest with a settling time of $91.9 \mathrm{sec}$.

\section{Baseline Tuning for the $S O(3) / 9$ Control Law}

Figure 5 shows the $\mathrm{SO}(3) / 9$ control law with actuator saturation. The saturation level is chosen to obtain a settling time of about $100 \mathrm{sec}$. 


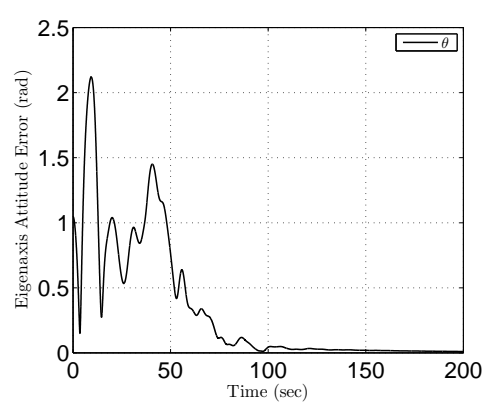

(a) Eigenaxis attitude error.

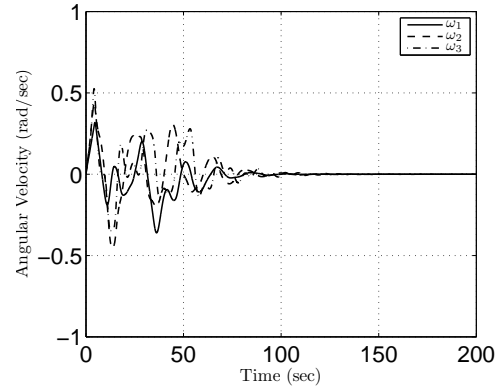

(b) Angular velocity components.

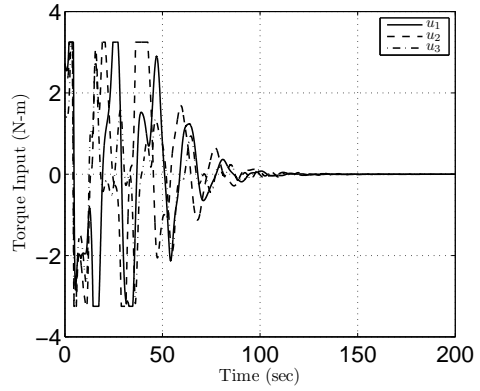

(c) Torque input.

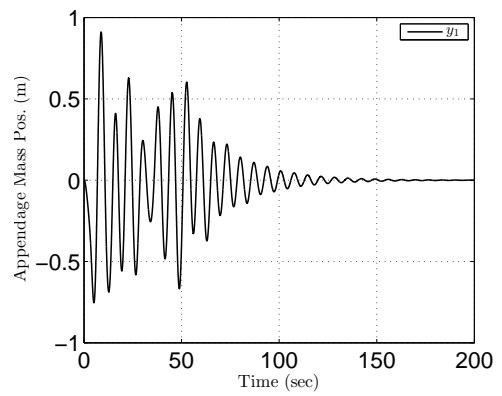

(d) Lumped mass position.

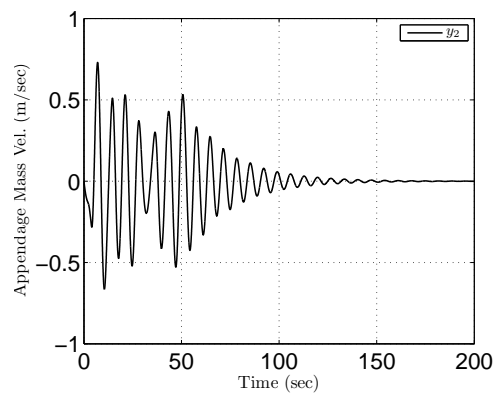

(e) Lumped mass velocity.

Figure 5: R2R for the $\mathrm{SO}(3) / 9$ controller with actuator saturation of $3.24 \mathrm{~N}-\mathrm{m}$. The maneuver is a 60 -deg rotation about the body-fixed frame direction $n=\frac{1}{\sqrt{3}}\left[\begin{array}{lll}1 & 1 & 1\end{array}\right]^{\mathrm{T}}$. The spacecraft reaches the desired attitude, and the lumped mass is brought to rest with a settling time of $95.0 \mathrm{sec}$.

\section{Baseline Tuning for RCAC for Saturation and Settling time}

For RCAC we define the performance variable

$$
z=\left[\begin{array}{l}
\tilde{\omega} \\
S
\end{array}\right],
$$

and we select the controller order $n_{c}=12$, the initial covariance parameter $\alpha(k)=0.1$, and the forgetting factor $\eta(0)=0$. Since the spacecraft dynamics are nonlinear, the Markov parameters used for RCAC are based on the rigid body spacecraft linearized model described in $^{24}$ where the first Markov parameter $\mathcal{H}_{1}$ of the linearized dynamics is given by

$$
\mathcal{H}_{1}=\left[\begin{array}{c}
h B \\
h^{2} B
\end{array}\right] \text {. }
$$

Figures 6 and 7 show the closed-loop performance for an illustrative R2R maneuver in the presence of actuator saturation of 0.12 and $0.015 \mathrm{~N}-\mathrm{m}$, respectively. 


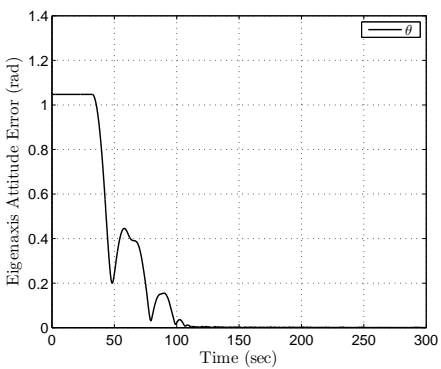

(a) Eigenaxis attitude error.

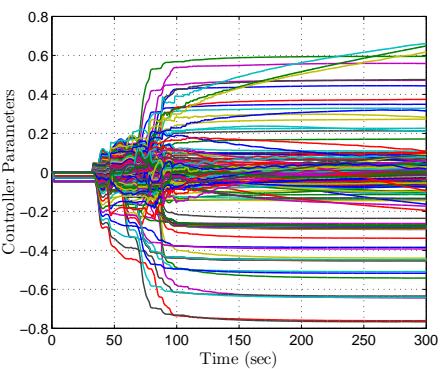

(d) Controller coefficients.

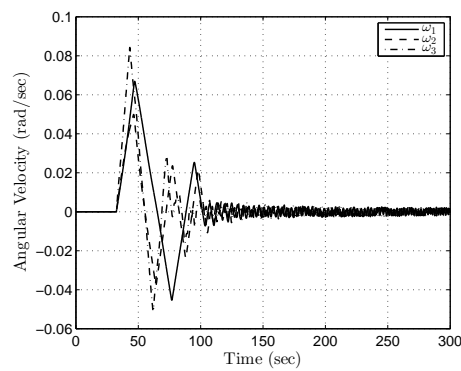

(b) Angular velocity components.

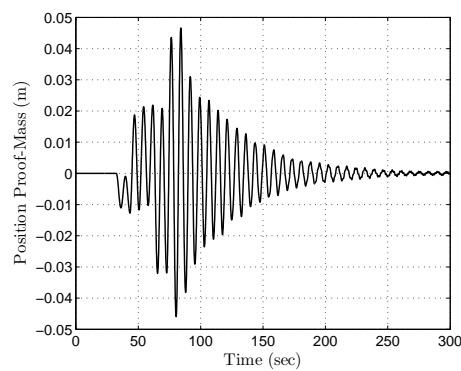

(e) Lumped mass position.

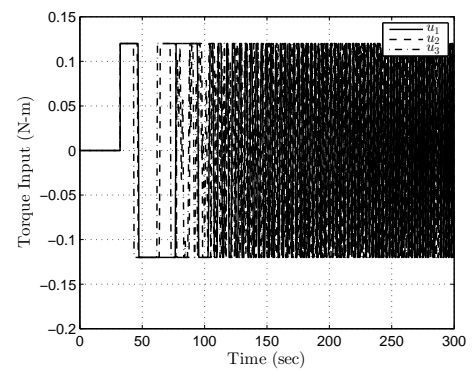

(c) Torque input.

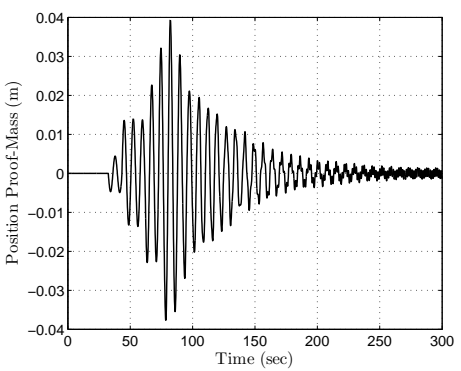

(f) Lumped mass velocity.

Figure 6: R2R for RCAC with actuator saturation of $0.12 \mathrm{~N}-\mathrm{m}$. The maneuver is a 60-deg rotation about the body-fixed frame direction $n=\frac{1}{\sqrt{3}}\left[\begin{array}{lll}1 & 1 & 1\end{array}\right]^{\mathrm{T}}$. The spacecraft reaches the desired attitude, and the lumped mass is brought to rest with a settling time of $102.9 \mathrm{sec}$.

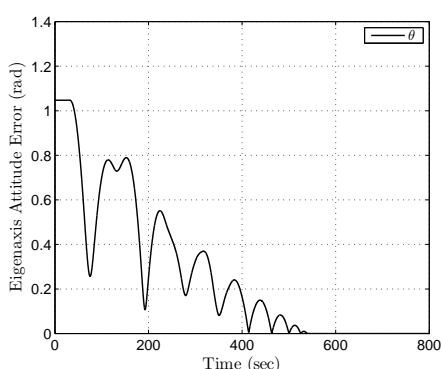

(a) Eigenaxis attitude error.

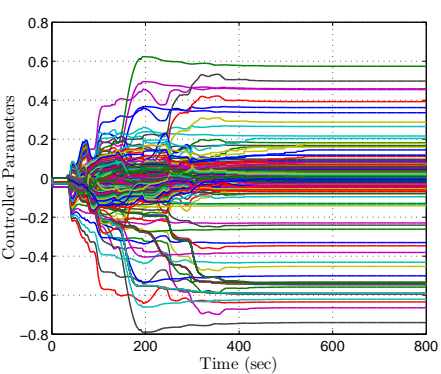

(d) Controller coefficients.

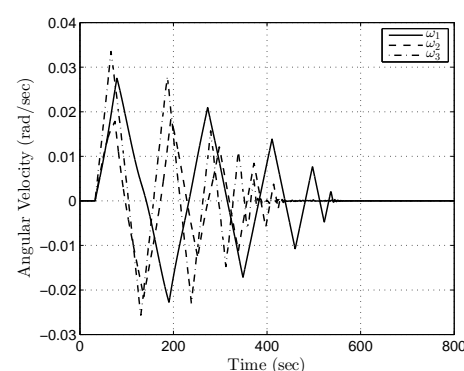

(b) Angular velocity components.

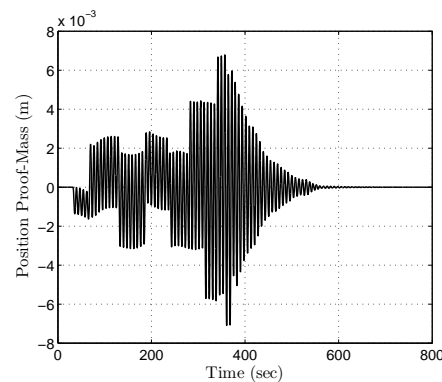

(e) Lumped mass position.

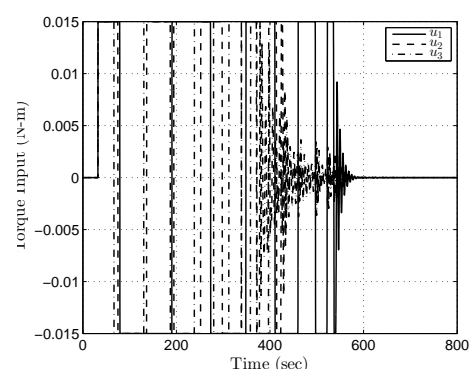

(c) Torque input.

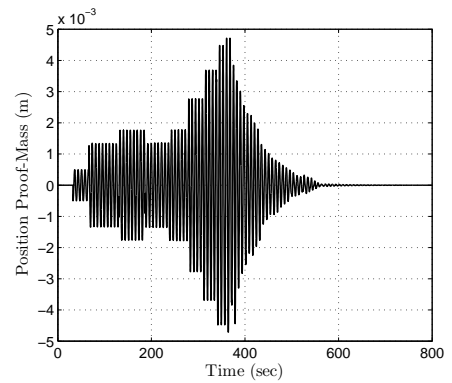

(f) Lumped mass velocity.

Figure 7: R2R for RCAC with actuator saturation of $0.015 \mathrm{~N}-\mathrm{m}$. The maneuver is a 60-deg rotation about the body-fixed frame direction $n=\frac{1}{\sqrt{3}}\left[\begin{array}{lll}1 & 1 & 1\end{array}\right]^{\mathrm{T}}$. The spacecraft reaches the desired attitude, and the lumped mass is brought to rest with a settling time of $503.2 \mathrm{sec}$. 


\section{Torque Saturation Variation}

In this section we study the performance robustness of the $\mathrm{SO}(3)$ control laws and RCAC under saturation levels and tuning parameters established in previous section. We represent the settling time as a function of different torque saturation levels, and define a criterion to consider actuator saturation for the robustness studies of sections VIII and IX.

\section{A. SO(3) Control Laws with Torque Saturation Variation}

We examine the performance of all four $\mathrm{SO}(3)$ controllers in the presence of torque saturation. Figure 8 shows the performance of the $\mathrm{SO}(3)$ controllers for $\mathrm{R} 2 \mathrm{R}$ with different torque saturation levels. Note that the settling time increases as the saturation level decreases. Figure 8 shows that there exist two distinct settling time ranges.

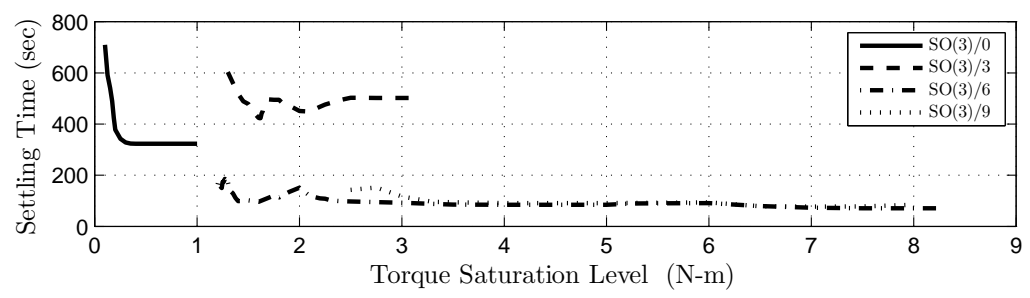

Figure 8: R2R settling time for the $\mathrm{SO}(3)$ control laws as a function of torque saturation level. The same saturation level is applied to each axis. Note that $\mathrm{SO}(3) / 0$ and $\mathrm{SO}(3) / 3$ have longer settling times than $\mathrm{SO}(3) / 6$ and $\mathrm{SO}(3) / 9$.

\section{B. RCAC with Torque Saturation Variation}

Figure 9 shows the settling time of RCAC as a function of the actuator saturation level.

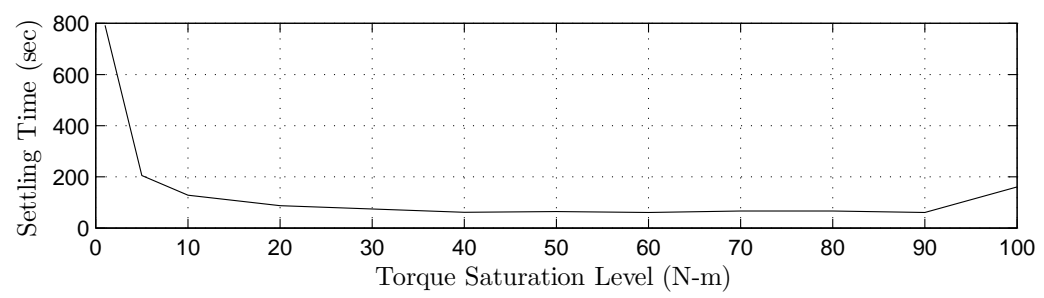

Figure 9: R2R settling time for RCAC as a function of torque saturation level. The same saturation level is applied to each axis.

\section{Baseline Saturation Specification}

Based on the results of the previous sections, we now define torque saturation levels for each controller that will be used for the rest of this paper. For the case where no saturation is applied, the $\mathrm{SO}(3) / 0$ and $\mathrm{SO}(3) / 3$ controllers exhibit settling times of about $500 \mathrm{sec}$, while the $\mathrm{SO}(3) / 6$ and $\mathrm{SO}(3) / 9$ controllers exhibit settling times of about 100 sec. We then choose saturation levels that provide settling times similar to the settling time obtained without saturation.

To compare RCAC with the SO(3) controllers, we consider two different torque saturation levels for this controller. The first level is defined as the saturation level for which RCAC exhibits settling times of $500 \mathrm{sec}(\mathrm{RCAC}(500))$, as well as the saturation level for which RCAC exhibits a settling time of $100 \mathrm{sec}(\mathrm{RCAC}(100))$. We will use RCAC(100) and $\mathrm{RCAC}(500)$ nomenclature in the next sections. 
Based on Figures 2-7, the saturation levels defined for $\mathrm{RCAC}(500)$ are $0.16 \mathrm{~N}-\mathrm{m}$ for $\mathrm{SO}(3) / 0,1.43 \mathrm{~N}-\mathrm{m}$ for $\mathrm{SO}(3) / 3$ and $0.12 \mathrm{~N}-\mathrm{m}$ for RCAC, and the saturation levels defined for RCAC(100) are $2.36 \mathrm{~N}-\mathrm{m}$ for $\mathrm{SO}(3) / 6,3.24 \mathrm{~N}-\mathrm{m}$ for $\mathrm{SO}(3) / 9$ and $0.015 \mathrm{~N}-\mathrm{m}$ for RCAC.

\section{Rigid Body Inertia Variation}

We now study robustness to uncertainty in the rigid body inertia. We define two different inertia variations. First we examine robustness to actuator misalignment relative to a body-fixed direction. Second we study robustness to changes in the components of the inertia matrix.

To model actuator misalignment we rotate the inertia matrix by the angle $\theta$ about the body-fixed direction $n$. The rotated inertia matrix $J^{\prime}$ is defined by

$$
J^{\prime} \triangleq \mathcal{R}_{J}(\theta)^{\mathrm{T}} J \mathcal{R}_{J}(\theta)
$$

We use Rodrigues's formula (56) to construct the rotation matrix $R_{J}(\theta)$. We rotate the inertia matrix $J$ by the angle $\theta \in\left(-180^{\circ}, 180^{\circ}\right]$ about the body-fixed direction $n=\frac{1}{\sqrt{3}}\left[\begin{array}{lll}1 & 1 & 1\end{array}\right]^{\mathrm{T}}$.

In the case of inertia variations, we examine the inertia matrix components variation between the nominal inertia cases defined below. Figure 10 shows the triangular region of feasible principal moments of inertia of a rigid body. There are five cases that are highlighted for the principal moments of inertia $\lambda_{1} \geq \lambda_{2} \geq \lambda_{3}>0$, where $\lambda_{1}, \lambda_{2}, \lambda_{3}$ satisfy the triangle inequality $\lambda_{1}<\lambda_{2}+\lambda_{3}$. Let $M$ denote the mass of the rigid body. The point $\lambda_{1}=\lambda_{2}=\lambda_{3}$ corresponds to a sphere of radius $R=\sqrt{\frac{5 \lambda_{1}}{2 M}}$; the point $\lambda_{1}=\lambda_{2}=2 \lambda_{3}$ corresponds to a cylinder of length $l$ and radius $r$, where $l / r=3$ and $r=\sqrt{\frac{2 \lambda_{1}}{M}}$; and the point $\lambda_{1}=\frac{6}{5} \lambda_{2}=2 \lambda_{3}$ is located at the centroid of the triangular region. The remaining cases in Figure 10 are limiting cases. As established in the baseline setup, $\lambda_{1}$ is normalized to $\lambda_{1}=30$ $\mathrm{kg}-\mathrm{m}^{2}$. we let $J_{1}, J_{2}, J_{3}, J_{4}, J_{5}$ correspond to the points noted in Figure 10 . These matrices, which correspond to the sphere, cylinder with $l / r=3$, centroid, thin disk, and thin cylinder, respectively, are defined as $J_{1}=\operatorname{diag}(30,30,30)$, $J_{2}=\operatorname{diag}(30,30,15), J_{3}=\operatorname{diag}(30,25,15), J_{4}=\operatorname{diag}(30,15,15)$, and $J_{5}=\operatorname{diag}(30,30,0.3)$. We vary the inertia by using a linear scaling between these points using

$$
J_{i j}(\alpha)=(1-\alpha) J_{i}+\alpha J_{j},
$$

where $\alpha \in[0,1]$ for $i, j \in\{(1,5),(3,1),(3,5),(3,4)\}$. 


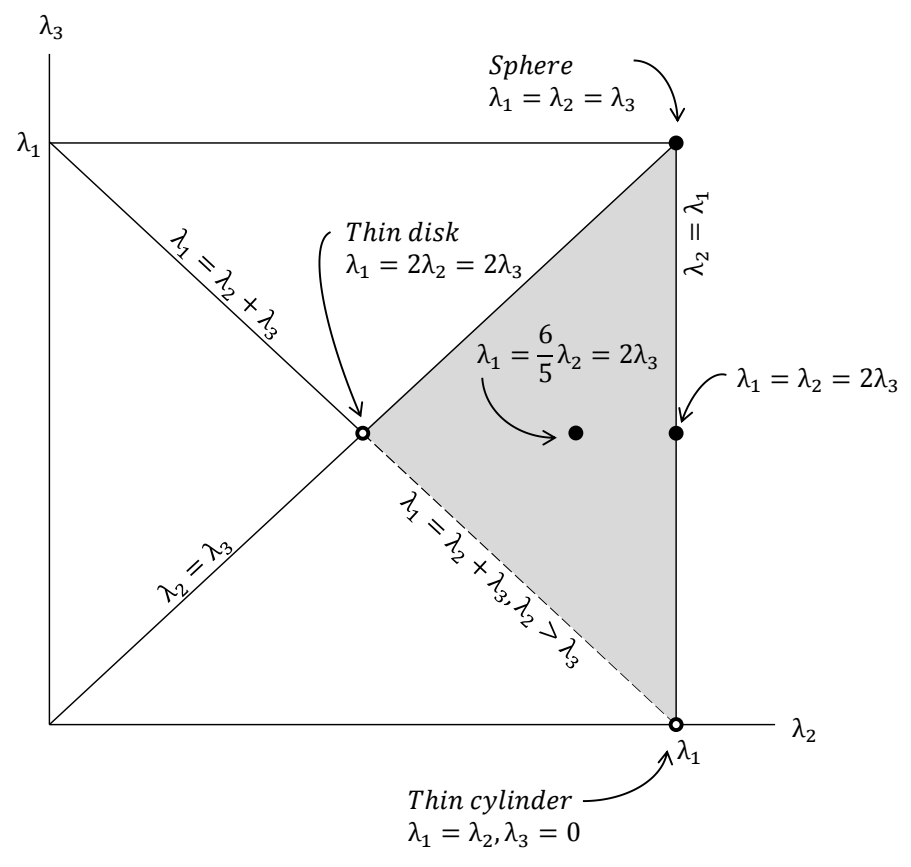

Figure 10: Feasible region of the principal moments of inertia $\lambda_{1}, \lambda_{2}, \lambda_{3}$ of a rigid body satisfying $0<\lambda_{3} \leq$ $\lambda_{2} \leq \lambda_{1}$, where $\lambda_{1}<\lambda_{2}+\lambda_{3}$. The shaded region shows all feasible values of $\lambda_{2}$ and $\lambda_{3}$ in terms of the largest principal moment of inertia $\lambda_{1}$. The open dots and dashed line segment indicate nonphysical, limiting cases.

\section{A. SO(3) Control Laws with Inertia Variation}

Figure 11 shows robustness to actuator misalignment for the $\mathrm{SO}(3)$ control laws. The controllers are able to bring the spacecraft to rest at the desired attitude despite off-diagonal terms in the inertia matrix. For RCAC(500), both controllers are able to bring the spacecraft to rest. However, $\mathrm{SO}(3) / 3$ is more sensitive to actuator misalignment than $\mathrm{SO}(3) / 0$. For RCAC(100), settling time remains unaffected for both controllers except for certain angles where the settling time increases substantially.

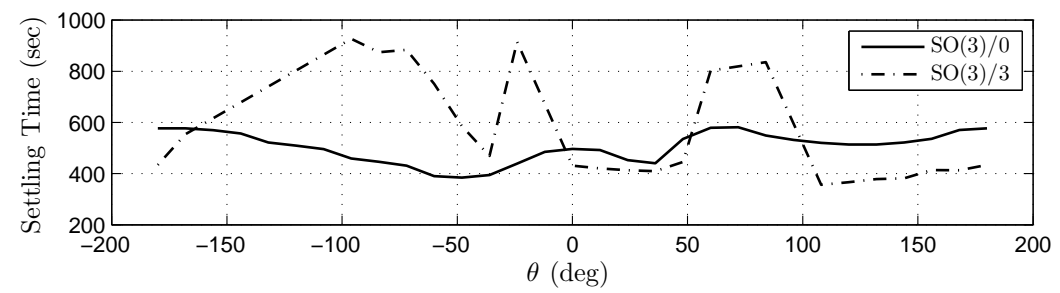

(a) $\mathrm{SO}(3) / 0$ and $\mathrm{SO}(3) / 3$ saturated as $\mathrm{RCAC}(500)$.

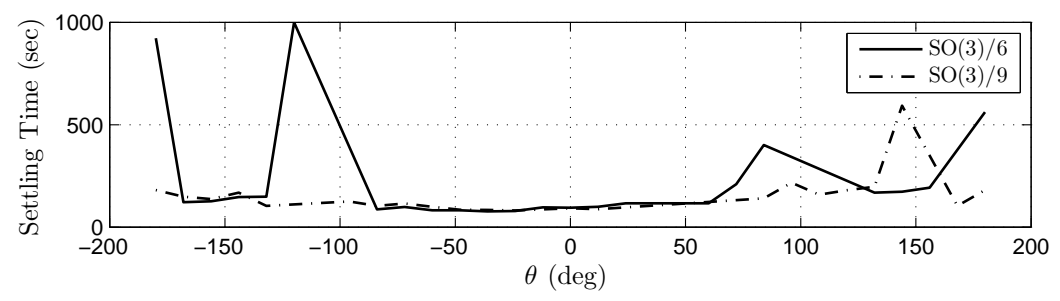

(b) $\mathrm{SO}(3) / 6$ and $\mathrm{SO}(3) / 9$ saturated as $\mathrm{RCAC}(100)$.

Figure 11: R2R settling time for the $\mathrm{SO}(3)$ control laws as a function of the principal-frame/body-frame rotation angle $\theta$ for a misalignment about the body-fixed direction $n=\frac{1}{\sqrt{3}}\left[\begin{array}{lll}1 & 1 & 1\end{array}\right]^{\mathrm{T}}$. 
Next, we show robustness for the $\mathrm{SO}(3)$ control laws to variations in the spacecraft inertia distribution as defined in (63). Figure 12 shows the settling time as a function of the scaling parameter $\alpha$ for $\operatorname{RCAC}(100)$. Note that when the inertia matrix is close to represent an inertia nominal shape (that is, cylinder, sphere, thin cylinder or thin disk) the controllers are not able to bring the spacecraft to rest.

Figure 13 shows the settling time variation as a function of the scaling parameter $\alpha$ for $\operatorname{RCAC}(500)$. These controllers seems to bring the spacecraft to rest for more $\alpha$ values than the controllers of $\operatorname{RCAC}(100)$. Note that the $\mathrm{SO}(3) / 0$ controller shows notable robustness with all four different inertia variations, and the $\mathrm{SO}(3) / 3$ controller actuation degrades when high $\alpha$ values are used.

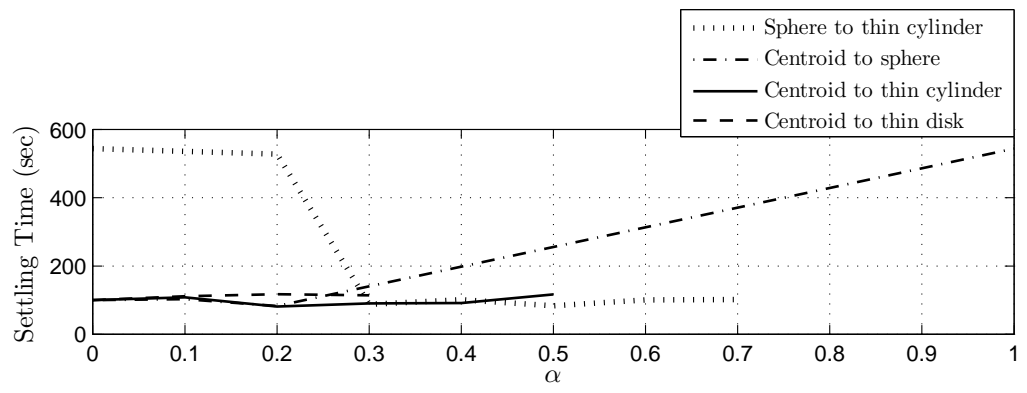

(a) $\mathrm{SO}(3) / 6$ saturated as $\mathrm{RCAC}(100)$.

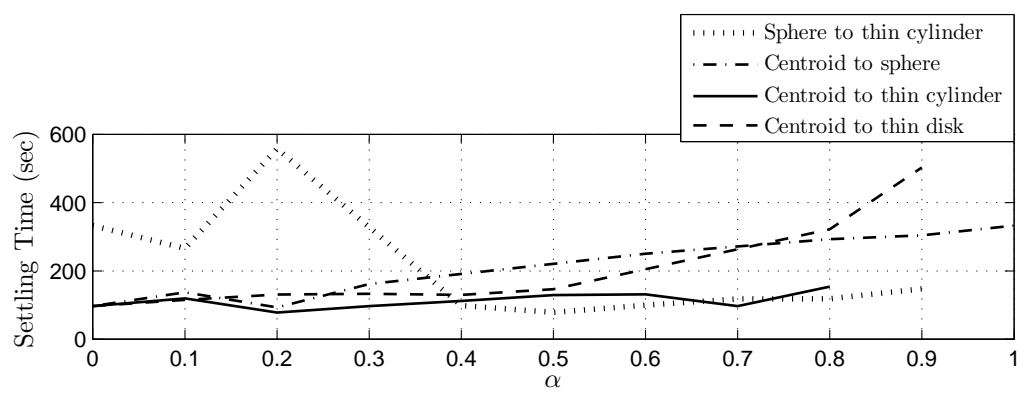

(b) $\mathrm{SO}(3) / 9$ saturated as $\mathrm{RCAC}(100)$.

Figure 12: R2R settling time for the $\mathrm{SO}(3) / 6$ and $\mathrm{SO}(3) / 9$ control laws as a function of the scaling parameter $\alpha$ for various combinations of inertia matrices. 


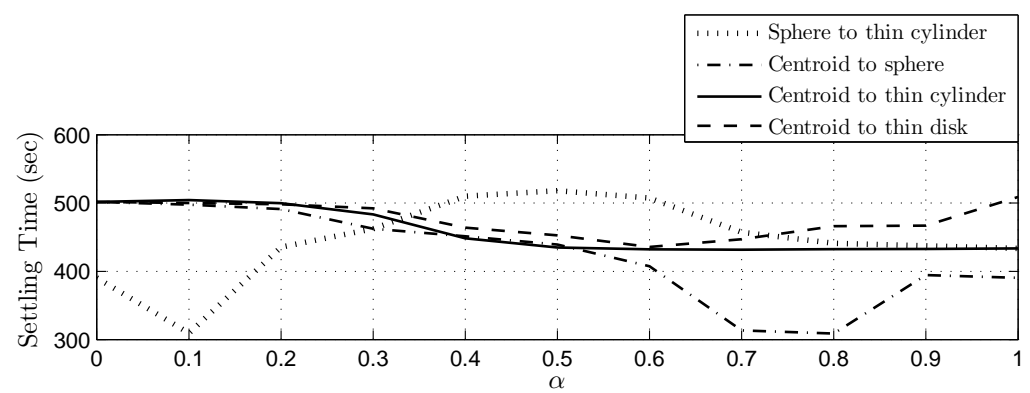

(a) $\mathrm{SO}(3) / 0$ saturated as $\mathrm{RCAC}(500)$.

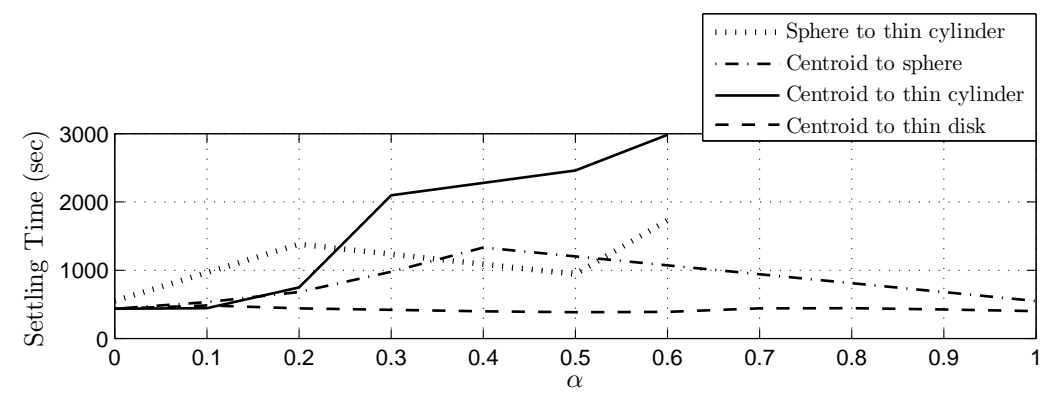

(b) $\mathrm{SO}(3) / 3$ saturated as $\mathrm{RCAC}(500)$.

Figure 13: R2R settling time for the $\mathrm{SO}(3) / 0$ and $\mathrm{SO}(3) / 3$ control laws as a function of the scaling parameter $\alpha$ for various combinations of inertia matrices.

\section{B. RCAC with Inertia Variation}

We study robustness for RCAC to actuator misalignment in Figure 14. For RCAC(500), the settling time increases by $44 \%$ in some cases. However, the controller brings the spacecraft to rest at the desired attitude despite the presence of off-diagonal inertia terms. Similarly, RCAC(100) is able to arrive to the desired attitude with settling time increasing by $71 \%$ in some cases.

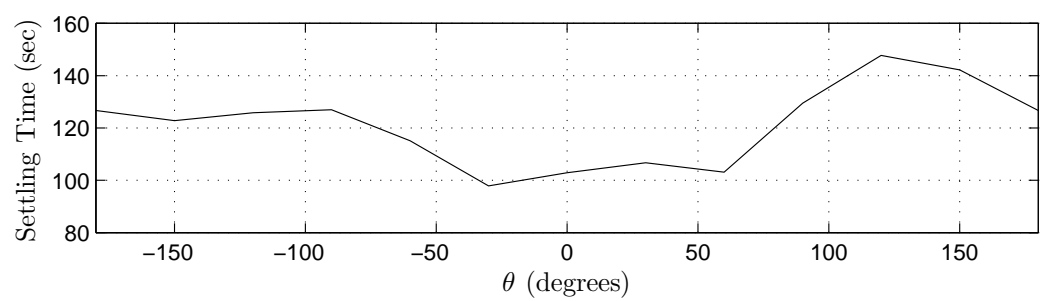

(a) RCAC saturated as RCAC(500).

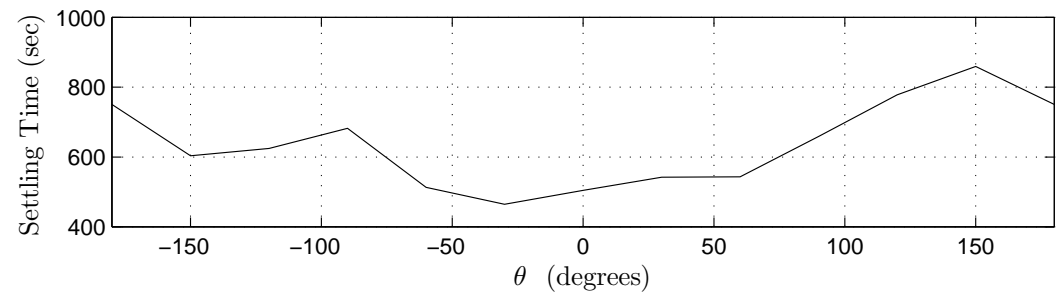

(b) RCAC saturated as RCAC(100).

Figure 14: R2R settling time for RCAC as a function of the scaling parameter $\alpha$ for various combinations of inertia matrices. 
Next, we show robustness for RCAC to variations in the spacecraft inertia distribution by varying the principal moments of inertia. Figure 15 shows the settling time as a function of the scaling parameter $\alpha$. Note that RCAC brings the spacecraft to rest for every inertia variation from both RCAC(500) and RCAC(100).

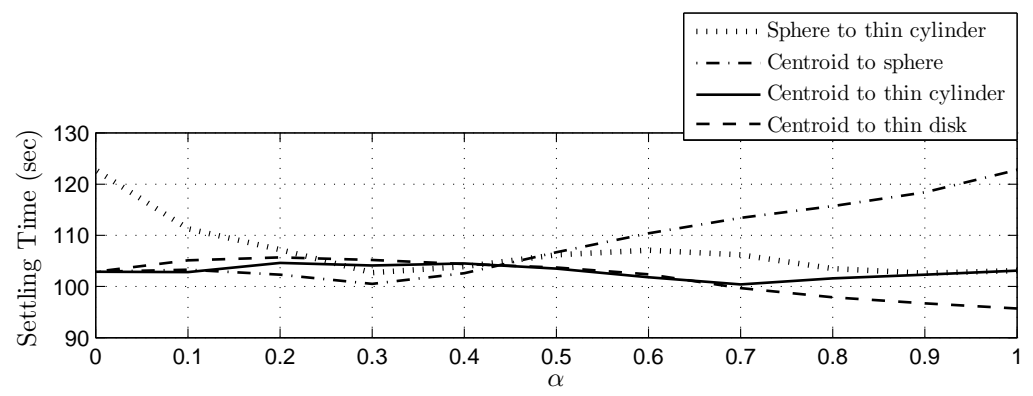

(a) RCAC saturated as RCAC(100).

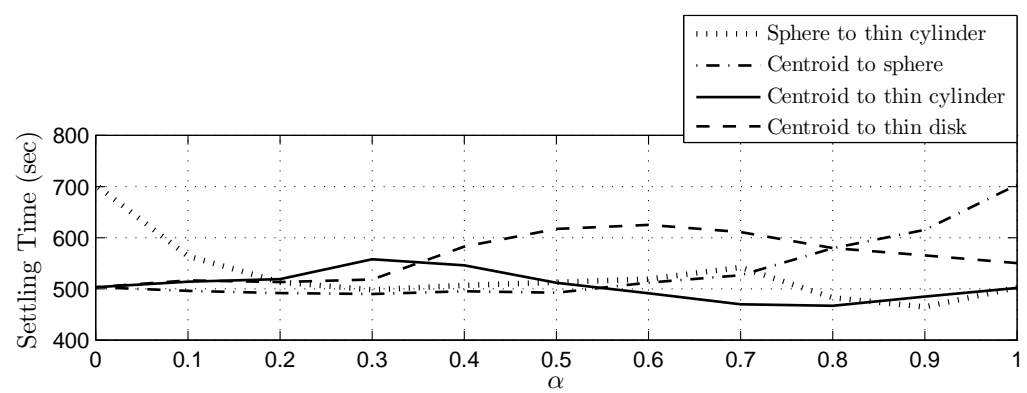

(b) RCAC saturated as RCAC(100).

Figure 15: R2R settling time for RCAC as a function of the scaling parameter $\alpha$ for various combinations of inertia matrices. The controller is robust to all inertia.

\section{Robustness to Flexible Mode Variation}

We now study robustness to variations in the unmodeled spacecraft flexible mode. These variations involve the inertia of the lumped mass and the spring stiffness. First we keep the spring stiffness constant at $k=2 \mathrm{~N}-\mathrm{m}$ while varying the lumped mass over $3 \leq m \leq 30 \mathrm{~kg}$. Then we maintain the mass constant at $m=3 \mathrm{~kg}$ while varying the spring stiffness over $0.01 \leq k \leq 100 \mathrm{~N}-\mathrm{m}$.

\section{A. SO(3) Control Laws with Flexible Mode Variation}

Figure 16 shows the settling time as a function of $m$ for the $\mathrm{SO}(3)$ control laws. $\mathrm{SO}(3) / 0$ is able to bring the spacecraft to rest. The controllers exhibit higher settling time as $m$ increases. $\mathrm{SO}(3) / 3$ is not able to bring the spacecraft to rest as the mass magnitude is varied in that range. Finally, $\mathrm{SO}(3) / 6$ and $\mathrm{SO}(3) / 9$ are not able to bring the spacecraft to the desired attitude for certain values of $m$.

Figure 17 compares the settling time as a function of the spring stiffness. Both $\mathrm{SO}(3) / 0$ and $\mathrm{SO}(3) / 3$ bring the spacecraft to rest, with $\mathrm{SO}(3) / 0$ exhibiting the best performance. On the other hand, $\mathrm{SO}(3) / 6$ and $\mathrm{SO}(3) / 9$ controllers show sensitivity to the stiffness variation, and the settling time increases considerably as the stiffness decreases. Note that $\mathrm{SO}(3) / 9$ is not able to bring the spacecraft to rest for values of the stiffness lower than $10^{-1} \mathrm{~N}-\mathrm{m}$. 


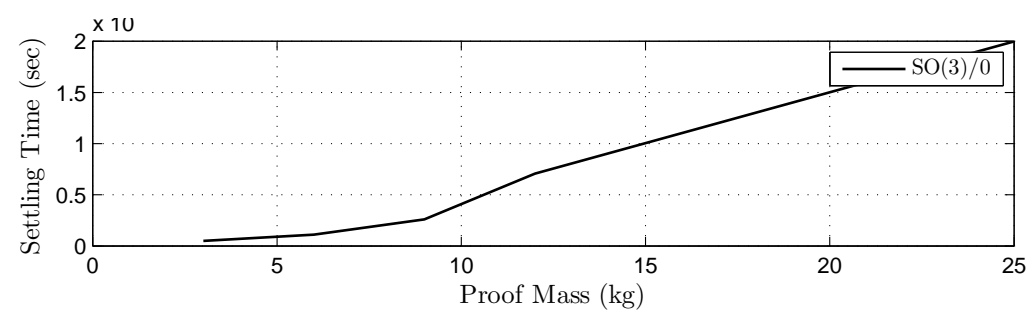

(a) $\mathrm{SO}(3) / 0$ saturated as $\mathrm{RCAC}(500)$.

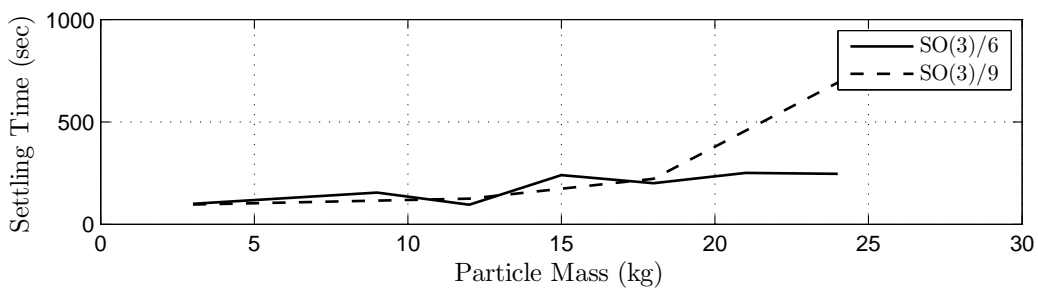

(b) $\mathrm{SO}(3) / 6$ and $\mathrm{SO}(3) / 9$ saturated as $\mathrm{RCAC}(100)$.

Figure 16: R2R settling time for the $\mathrm{SO}(3)$ control laws as a function of the lumped mass $m$ while the spring stiffness remains at its nominal value.

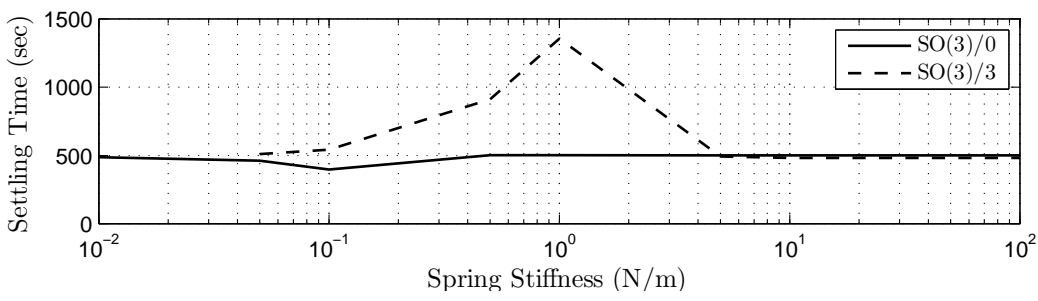

(a) $\mathrm{SO}(3) / 0$ and $\mathrm{SO}(3) / 3$ saturated as $\mathrm{RCAC}(500)$.

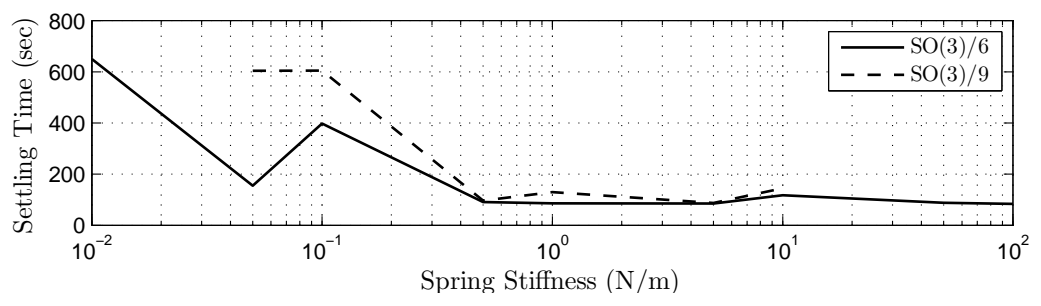

(b) $\mathrm{SO}(3) / 6$ and $\mathrm{SO}(3) / 9$ saturated as $\mathrm{RCAC}(100)$.

Figure 17: R2R settling time for the $\mathrm{SO}(3)$ control laws as a function of the spring stiffness while the lumped mass $m$ remains at its nominal value.

\section{B. RCAC with Flexible Mode Variation}

Figure 18 and 19 show the settling time as a function of the lumped mass $m$ for RCAC. Note that the settling time keeps within similar values for all cases studied. 


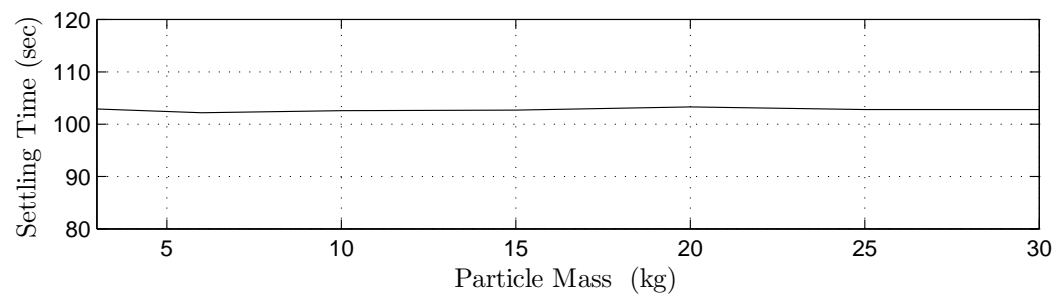

(a) RCAC saturated as $\mathrm{RCAC}(500)$.

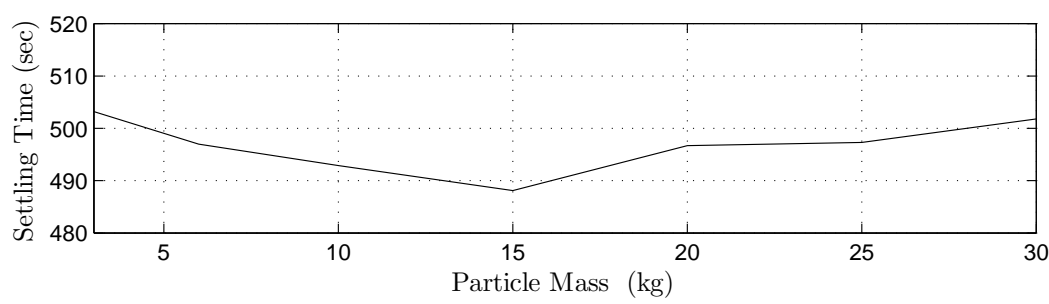

(b) RCAC saturated as $\mathrm{RCAC}(100)$.

Figure 18: R2R settling time for RCAC as a function of the particle mass while the spring stiffness remains at is nominal value.

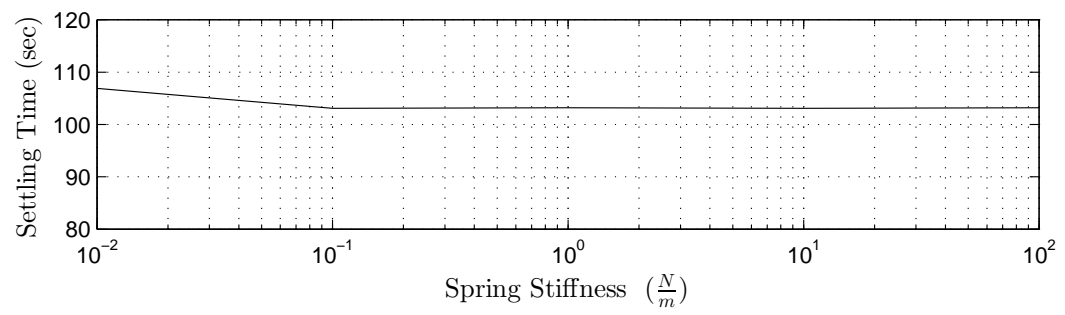

(a) RCAC saturated as RCAC(500).

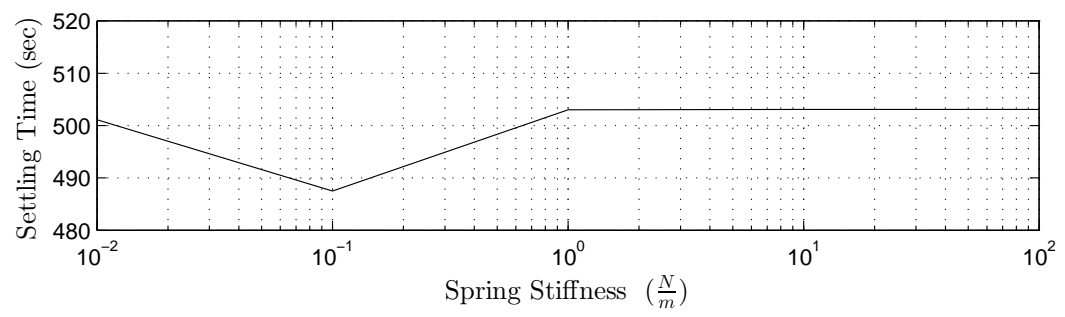

(b) RCAC saturated as RCAC(100).

Figure 19: R2R settling time for RCAC as a function of the spring stiffness while the lumped mass $m$ remains at its nominal value.

To further demonstrate RCAC robustness, we study settling time as a function of lumped mass and spring stiffness variations within a motion-to-rest (M2R) scenario. Now, we keep the baseline setup and define the new initial angular velocity $\omega_{0}=\left[\begin{array}{lll}0.5 & 0.5 & 0.5\end{array}\right]^{\mathrm{T}} \mathrm{rad} / \mathrm{sec}$. $\mathrm{RCAC}(500)$ and $\mathrm{RCAC}(100)$ criteria are maintained.

Figures 20 and 21 show that RCAC is able to bring the spacecraft to the desired attitude and angular velocity. The lumped mass motion is reduced to zero. Note that higher angular velocity leads to larger flexibility effects. 


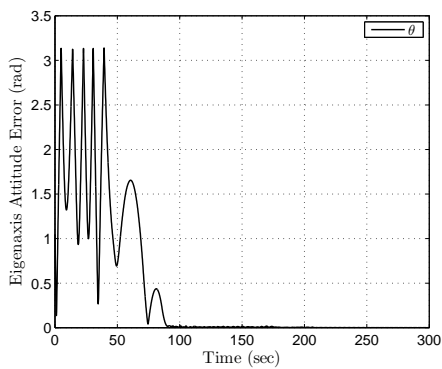

(a) Eigenaxis attitude error.

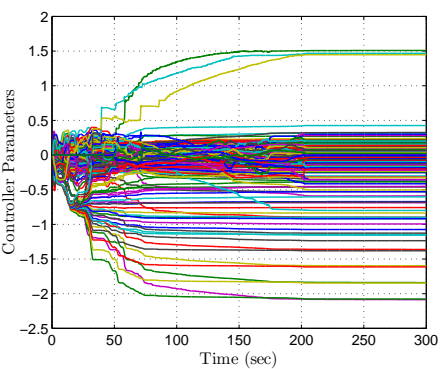

(d) Controller coefficients.

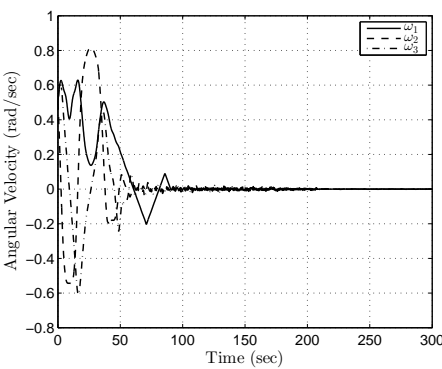

(b) Angular velocity components.

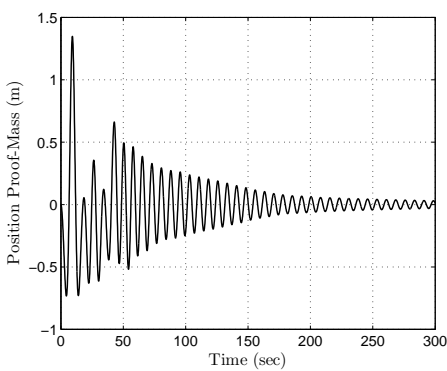

(e) Lumped mass position.

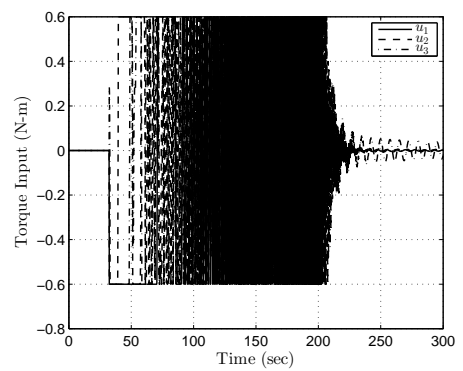

(c) Torque input.

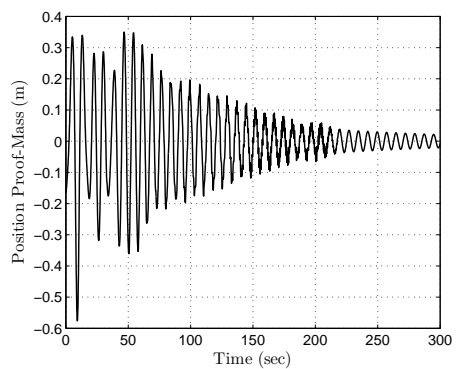

(f) Lumped mass velocity.

Figure 20: M2R settling time for RCAC with torque saturation at $0.6 \mathrm{~N}-\mathrm{m}$. The maneuver is a $60-\mathrm{deg}$ rotation about the body-fixed direction $n=\frac{1}{\sqrt{3}}\left[\begin{array}{lll}1 & 1 & 1\end{array}\right]^{\mathrm{T}}$. The settling time needed is $98.5 \mathrm{sec}$.

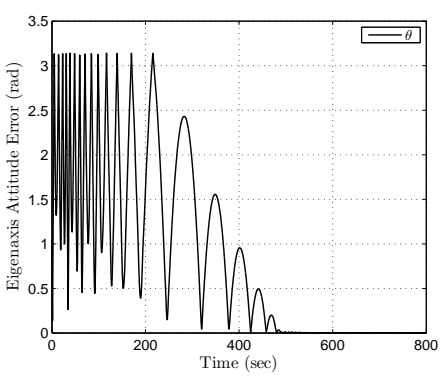

(a) Eigenaxis attitude error.

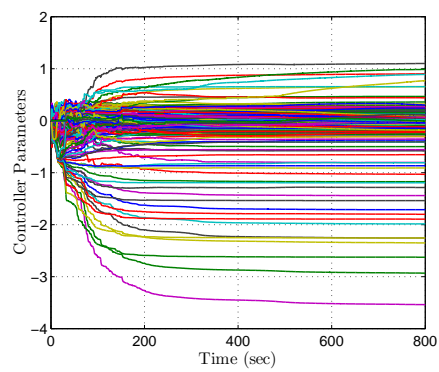

(d) Controller coefficients.

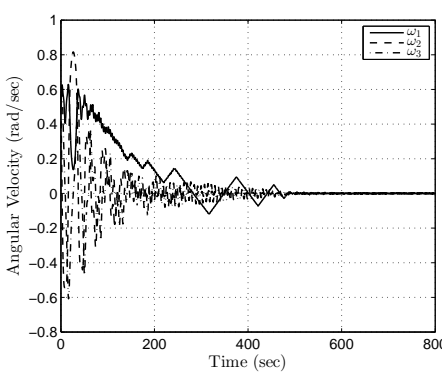

(b) Angular velocity components.

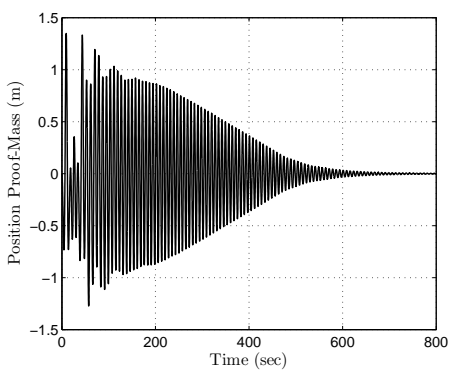

(e) Lumped mass position.

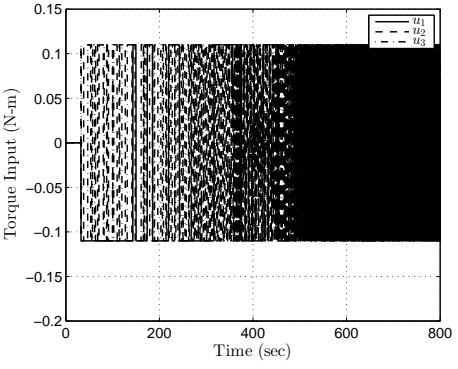

(c) Torque input.

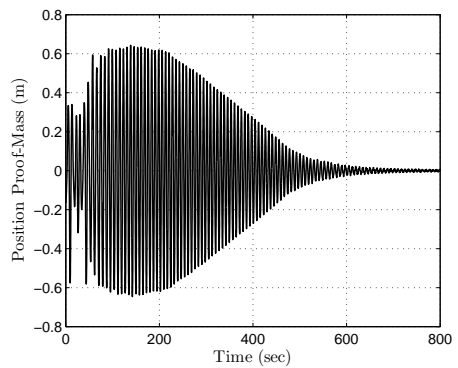

(f) Lumped mass velocity.

Figure 21: M2R settling time for RCAC with torque saturation at $0.11 \mathrm{~N}-\mathrm{m}$. The maneuver is a 60-deg rotation about the body-fixed direction $n=\frac{1}{\sqrt{3}}\left[\begin{array}{lll}1 & 1 & 1\end{array}\right]^{\mathrm{T}}$. The settling time needed is $488.1 \mathrm{sec}$.

Figures 22 and 23 represent the settling time as a function of the lumped mass $m$ and spring stiffness $k$ in a M2R 
scenario. The desired attitude is achieved for every simulation. However, the settling time increases notably compared with the R2R scenario.

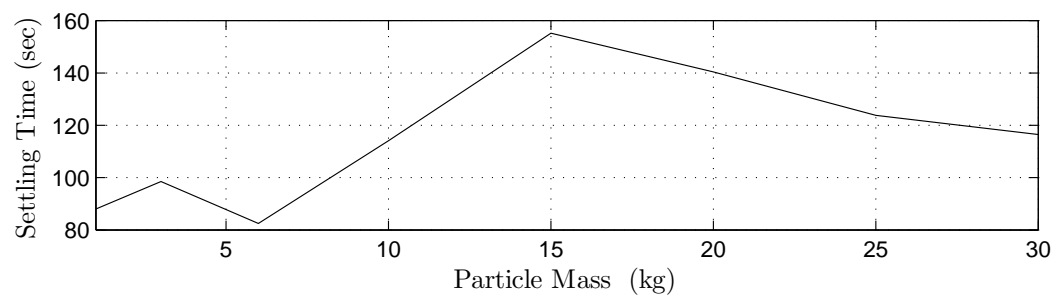

(a) RCAC saturated as $\mathrm{RCAC}(500)$.

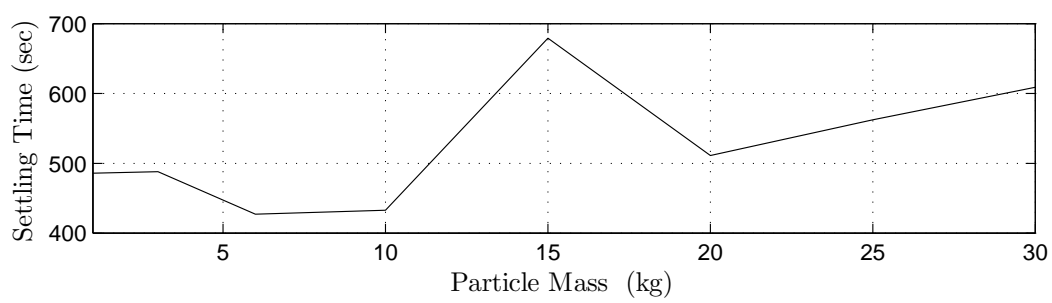

(b) RCAC saturated as RCAC(100).

Figure 22: M2R settling time for RCAC as a function of the particle mass while the spring stiffness remains at its nominal value. The spacecraft is initially at motion with $\omega_{0}=\left[\begin{array}{lll}0.5 & 0.5 & 0.5\end{array}\right]^{\mathrm{T}} \mathrm{rad} / \mathrm{sec}$. Note that settling time increases with specific lumped mass magnitudes.

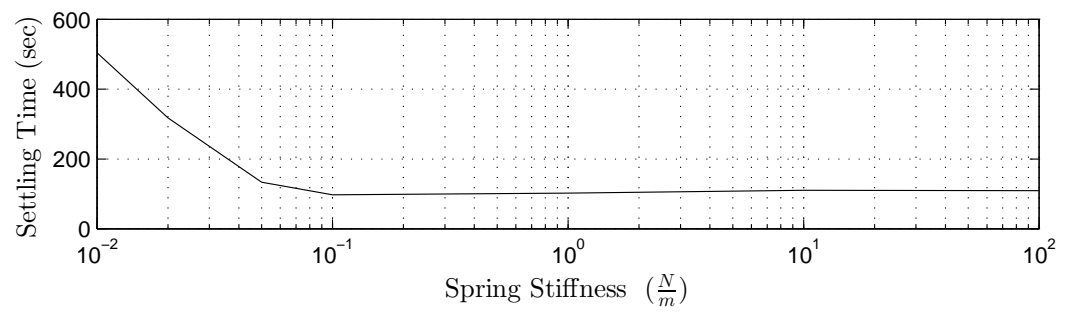

(a) RCAC saturated as $\mathrm{RCAC}(500)$.

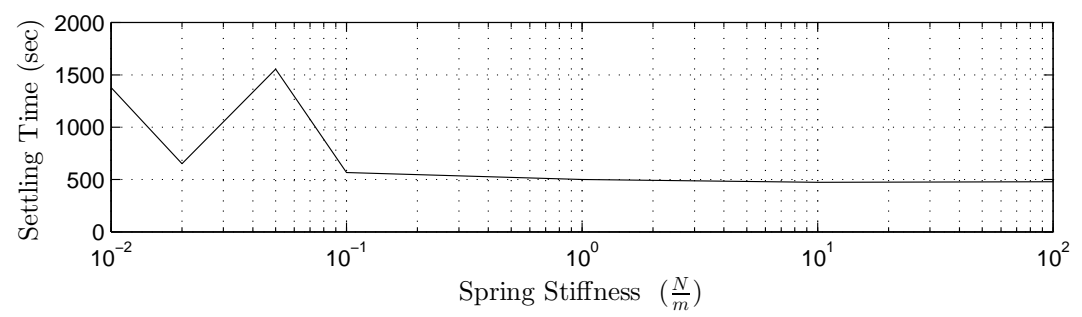

(b) RCAC saturated as RCAC(100).

Figure 23: M2R settling time for RCAC as a function of the spring stiffness while the lumped mass remains at its nominal value. The spacecraft is initially at motion with $\omega_{0}=\left[\begin{array}{lll}0.5 & 0.5 & 0.5\end{array}\right]^{\mathrm{T}} \mathrm{rad} / \mathrm{sec}$. Note that settling time increases for low stiffness values. 


\section{Conclusion}

We have compared the performance of several inertial free attitude control laws as applied to a spacecraft with a discrete flexible mode. RCAC presents a better performance than the $\mathrm{SO}(3)$ controllers for more restrictive saturation levels, with $\mathrm{SO}(3) / 0$ being the only one with similar performance at low saturation levels. Conversely, RCAC required low saturation levels to achieve the desired attitude.

The numerical results show that both $\mathrm{RCAC}$ and the $\mathrm{SO}(3)$ controllers are robust to actuator misalignment and variation of the inertia matrix. In general RCAC presents better performance than the $\mathrm{SO}(3)$ controllers, again with $\mathrm{SO}(3) / 0$ presenting the smaller variation in settling time criteria out of the $\mathrm{SO}(3)$ controllers. Finally, both controllers show robustness on the variation of flexible mode parameters, with RCAC outperforming again the SO(3) controllers for both change in the lumped mass and the stiffness. In general both control laws have demonstrated their robustness to unknown actuator saturation and plant uncertainties, which is a desired property to avoid the degradation of the accuracy of attitude control systems when structural flexibility of spacecraft comes into play.

\section{References}

${ }^{1}$ Wie, B., Space Vehicle Dynamics and Control, AIAA, 2nd ed., August 2008.

${ }^{2}$ Hughes, P., Spacecraft Attitude Dynamics, Dover Publications, 1st ed., December 2004.

${ }^{3}$ Kelkar, A. and Joshi, S. M., Control of Nonlinear Multibody Flexible Space Structures, Springer, 1st ed., August 1996.

${ }^{4}$ Junkins, J. and Kim, Y., Introduction to Dynamics and Control of Flexible Structures, AIAA, 1st ed., 1993.

${ }^{5}$ Meirovitch, L., Dynamics and Control of Structures, Wiley-Interscience, 1st ed., March 1990.

${ }^{6}$ Ahmed, J., Coppola, V. T., and Bernstein, D. S., “Asymptotic Tracking of Spacecraft Attitude Motion with Inertia Identification,” AIAA J. Guid. Contr. Dyn., Vol. 21, 1998, pp. 684-691.

${ }^{7}$ Egeland, O. and Godhavn, J.-M., "Passivity-Based Adaptive Attitude Control of a Rigid Spacecraft," IEEE Transactions on Automatic Control, Vol. 39, 1994, pp. 842-846. 1162.

${ }^{8}$ Wen, J. T.-Y. and Kreutz-Delgado, K., "The Attitude Control Problem," IEEE Transactions on Automatic Control, Vol. 36, 1991, pp. 1148-

${ }^{9}$ Junkins, J. L., Akella, M. R., and Robinett, R. D., "Nonlinear Adaptive Control of Spacecraft Maneuvers," AIAA J. Guid. Contr. Dyn., Vol. 20, 1997, pp. 1104-1110.

${ }^{10}$ Venugopal, R. and Bernstein, D. S., “Adaptive Disturbance Rejection Using ARMARKOV System Representations,” IEEE Trans. Contr. Sys. Tech., Vol. 8, 2000, pp. 257-269.

${ }^{11}$ Santillo, M. A. and Bernstein, D. S., "Adaptive Control Based on Retrospective Cost Optimization," AIAA J. Guid. Contr. Dyn., Vol. 33, 2010, pp. 289-304.

${ }^{12}$ D'Amato, A. M., Sumer, E. D., and Bernstein, D. S., "Frequency-Domain Stability Analysis of Retrospective-Cost Adaptive Control for Systems with Unknown Nonminimum-Phase Zeros," Proc. Conf. Dec. Contr., Orlando, FL, December 2011, pp. 1098-1103.

${ }^{13}$ Hoagg, J. B. and Bernstein, D. S., "Retrospective Cost Model Reference Adaptive Control for Nonminimum-Phase Systems," AIAA J. Guid. Contr. Dyn., Vol. 35, 2012, pp. 1767-1786.

${ }^{14}$ Sanyal, A. K., Lee, T., Leok, M., and McClamroch, N. H., "Global Optimal Attitude Estimation Using Uncertainty Ellipsoids,” Sys. Contr. Lett., Vol. 57, 2008, pp. 236-245.

${ }^{15}$ Lee, T., Computational Geometric Mechanics and Control of Rigid Bodies, Ph.D. thesis, University of Michigan, 2008.

${ }^{16}$ Lee, T., Leok, M., and McClamroch, N. H., "Lagrangian Mechanics and Variational Integrators on Two-Spheres," International Journal for Numerical Methods in Engineering, Vol. 79, No. 9, 2009, pp. 1147-1174.

${ }^{17}$ Chaturvedi, N. A., Global Dynamics and Stabilization of Rigid Body Attitude Systems, Ph.D. thesis, University of Michigan, Ann Arbor, MI, 2007.

${ }^{18}$ Sanyal, A., Fosbury, A., Chaturvedi, N., and Bernstein, D. S., "Inertia-Free Spacecraft Attitude Tracking with Disturbance Rejection and Almost Global Stabilization,” AIAA J. Guid. Contr. Dyn., Vol. 32, 2009, pp. 1167-1178.

${ }^{19}$ Cruz, G., Yang, X., Weiss, A., Kolmanovsky, I., and Bernstein, D. S., “Torque-saturated, Inertia-free Spacecraft Attitude Control,” Proc. AIAA Guid. Nav. Contr. Conf., Portland, OR, August 2011, AIAA-2011-6507.

${ }^{20}$ Weiss, A., Yang, X., Kolmanovsky, I., and Bernstein, D. S., "Inertia-Free Spacecraft Attitude Control with Reaction-Wheel Actuation," Proc. AIAA Guid. Nav. Contr. Conf., Toronto, August 2010, AIAA-2010-8297-163.

${ }^{21}$ Venugopal, R. and Bernstein, D., “Adaptive Disturbance Rejection Using ARMARKOV System Representations," IEEE Trans. Contr. Sys. Tech., Vol. 8, 2000, pp. 257-269.

${ }^{22}$ A. M. D'Amato, E. D. S. and Bernstein, D. S., "Frequency Domain Stability Analysis of Retrospective-Cost Adaptive Control for Systems with Unknown Nonminimum-Phase Zeros," Proc. Conf. Dec. Contr, Orlando, FL, December 2011, pp. 1098-1103.

${ }^{23}$ Hoagg, J. B. and Bernstein, D. S., "Retrospective Cost Model Reference Adaptive Control for Nonminimum-Phase Systems," AIAA J. Guid. Contr. Dyn., Vol. 35, 2012, pp. 1767-1786.

${ }^{24}$ G. Cruz, A. M. D. and Bernstein, D. S., "Retrospective Cost Adaptive Control of Spacecraft Attitude," Proc. AIAA Guid. Nav. Contr. Conf., Minneapolis, MN, August 2012, AIAA-2012-4624-236. 